\title{
Long-term change of meiofaunal species composition in a sandy beach, with description of 7 new species of Platyhelminthes
}

Werner Armonies ${ }^{*}$ iD

\begin{abstract}
Climate change and species introductions strongly changed macrobenthic community composition in the North Sea during past decades. To find out whether there was a similar change in meiofauna, I re-examined a semi-exposed sandy beach of the Island of Sylt that had been intensively studied between 1965 and 1975. These previous studies provide the baseline for temporal change. As before, the analysis of species composition focused on Platyhelminthes. Minor change in the physical properties of the beach during the past decades was compensated for by including two adjoining beaches, one slightly more sheltered and the other slightly more exposed. In due consideration of somewhat differing sample size, platyhelminth species richness barely changed over the past 50 years, and the numerical dominant species were still the same. However, roughly $10 \%$ of the 220 species found during this study were not previously recorded from the Island of Sylt. Thirteen are new to science though unpublished records indicate that half of them already occurred previously. Seven species described from elsewhere were recorded for the first time in the Sylt area. Based on their known geographical range, none of these can be unambiguously assigned an immigrant or introduced species. I conclude that (1) the platyhelminth fauna displayed high temporal stability over the past 50 years, and (2) though this beach is among the best studied in the world, a rate of 10\% new species indicates our knowledge on the diverse meiofauna is still relatively poor. Seven new species of Platyhelminthes are described and three re-described: Coelogynopora multispina n. sp., Pseudostomum gracilis Westblad 1955, Nesite ptychoperoides n. gen. n. sp., Haplovejdovskya scilliensis Faubel \& Warwick 2005, Haloplanella magnarhabdita n. sp., Haloplanella spiralis n. sp., Moevenbergia oculifagi n. sp., Coronhelmis inaequalis n. sp., Proxenetes inflatus den Hartog 1966, and Diascorhynchus falconis n. sp.
\end{abstract}

Keywords: Meiofauna, Platyhelminthes, Sandy beach, Diversity, Long-term change

\section{Background}

Climate change and introductions of non-native species strongly modified the North Sea benthos communities during the past decades $[13,42]$ and further change is expected [81]. However, this evidence only comes from larger-sized flora and fauna, while studies on small-sized organisms are scarce although they may constitute most of the biological diversity in these shallow waters, play an important role in food webs, and are sensitive to a wide

*Correspondence: werner.armonies@awi.de

Alfred-Wegener-Institut Helmholtz-Zentrum für Polar- und

Meeresforschung, Wattenmeerstation Sylt, Hafenstr. 43, 25992 List, Germany range of physical factors, including those associated with climate change [88]. Thus, change in the meiofaunal communities might affect ecosystems as strong as changes in other compartments. Nevertheless, meiofauna has widely been disregarded in studies related to climate change effects, presumably because of the high effort associated with sorting and species determination of small fauna and the lack of baseline data.

The beach called 'Hausstrand' or 'beach near the old Litoralstation' on the island of Sylt is an exception. This semi-exposed beach was intensively studied for meiofauna between 1965 and 1975, starting with Schmidt $[68,69]$ who described the physical factors and gave a 
general overview on meiofaunal abundance. In the following years individual taxa were studied one by one in detail (Table 1) and most of these studies adhered to a sampling protocol developed by Schmidt [68]. Altogether some 50,000 samples were collected and $>1$ million individuals counted and identified, which gave a total of 652 meiofaunal species [3]. Platyhelminths were richest in species, but this may be due to the high intensity of studies on that taxon (Table 1). In total, these studies may serve as a baseline for comparisons of the meiofaunal community in the late 1960s and the 1970s with future studies, with the best quality of baseline data for the taxon Platyhelminthes.

Numerically the 'Hausstrand' meiofauna is dominated by harpacticoid copepods in the steep-angled upper beach face with coarser sediment while nematodes dominate in abundance in the lower intertidal flats with finer sediment [2], as is typical for meiofaunal taxonomic composition in habitats like this (e.g., [16]. Only occasionally are Platyhelminthes among the numerical dominant meiofaunal taxa, e.g., in the most turbulent zone of wave

Table 1 Major studies on meiofaunal taxa at 'Hausstrand', Island of Sylt, North Sea

\begin{tabular}{|c|c|}
\hline Taxon & Authors \\
\hline Ciliata & Hartwig $[34,35]$ \\
\hline Cnidaria & Schmidt [69] \\
\hline Acoelomorpha & Dörjes [17, 18], Faubel $[25,28,29]$ \\
\hline Gnathostomulida & Müller and Ax [55] \\
\hline \multicolumn{2}{|l|}{ Plathelminthes } \\
\hline Catenulida & Faubel [27] \\
\hline Macrostomida & Pawlak [57], Faubel $[26,28]$ \\
\hline Proseriata & Sopott $[73,74]$ \\
\hline Typhloplanoida & Ax and Heller [8], Ax [6], Ehlers [19-21] \\
\hline Kalyptorhynchia & Ax and Heller [8], Schilke [67], Hoxhold [39] \\
\hline Dalyellioida & Ax [5], Ehlers [20] \\
\hline \multicolumn{2}{|l|}{ Gastrotricha } \\
\hline Macrodasyoida & Schmidt and Teuchert [71], Potel and Reise [58] \\
\hline Chaetonotoida & Schmidt and Teuchert $[52,71]$ \\
\hline Rotatoria & Tzschaschel $[78,79]$ \\
\hline Nematoda & Blome [10-12] \\
\hline Nemertini & $\operatorname{Mock}[51,53]$ \\
\hline Polychaeta & $\begin{array}{l}\text { Westheide [83-86], Schmidt [69, 70], Meineke and } \\
\text { Westheide [46], Mock [54] }\end{array}$ \\
\hline Oligochaeta & Kossmagk-Stephan [41] \\
\hline Tardigrada & Schmidt [69] \\
\hline Copepoda & Mielke [48-50] \\
\hline Ostracoda & Schmidt [69] \\
\hline \multicolumn{2}{|l|}{ Acari } \\
\hline Halacaridae & Bartsch and Schmidt [9] \\
\hline
\end{tabular}

Taxonomic subdivisions according to the surveys cited exposed beaches [2], and Remane [60] coined the term 'Otoplana-zone' for this part of exposed beaches, after the plathelminth taxon Otoplanidae. But ecologically Platyhelminths may be more important than expected from abundance, because the majority of species seem to be predators, mostly feeding on other meiofaunal specimens (e.g., [45].

Long-term comparisons of species composition need to account for habitat change, in particular in shallow coastal areas. For 'Hausstrand', Schmidt [68] described the beach morphology and sediment composition in the mid-sixties, but gave no information on spatial and temporal variability. Therefore we can only state that sediment composition is still within the ranges described previously. However, beach morphology changed. The reflective beach face (i.e. the upper part of the beach with a steep angle) has still the same extension and angle but next to the ferry landing, its position shifted by some $20 \mathrm{~m}$ seawards. At the same time, the dissipative sandflat (i.e. the lower part of the beach increasing in height by a very small angle) became much smaller. Schmidt [68] described it to be $100 \mathrm{~m}$ wide (bend to mean low tide level), but in $2015 \mathrm{I}$ only measured $60-70 \mathrm{~m}$. This is due to increasing erosion of the adjoining tidal gully, which is not a local phenomenon but part of long-term changes that already started in medieval times and are nowadays strengthened by sea level rise [37, 63]. As a consequence, the smaller extension of the dissipative sandflat may provide less shelter from waves for the reflective beach face, potentially changing wave exposition and improving living conditions for some species and deteriorating them for others. Thus, gains and losses of species from the previous species spectrum may partly be due to habitat dynamics. To compensate for that, two adjoining beaches were studied for comparison, one with a slightly higher and the other with a slightly lower degree of exposition to waves. Accordingly, sediment composition in these beaches was slightly coarser or finer, respectively, than in 'Hausstrand.'

In the 1960s and 1970s, 168 species of platyhelminths had been found in Hausstrand. This high number is based on several studies (Table 1) with a high number of samples. In this study sampling intensity is lower by about one order of magnitude. Therefore, I a priori expected to re-detect less species than had been found previously. On the other hand, detecting species today that had not been detected before despite of the high previous sampling intensity is assumed to indicate either habitat change, or change in the local faunal composition as may be brought about by immigration or anthropogenic translocation of species. Therefore, main emphasis in this study is on species composition, with special attention to species not recorded before. 


\section{Methods}

The study sites are all located on the sheltered side of the barrier island of Sylt in the eastern North Sea $\left(55^{\circ} \mathrm{N}\right.$, $\left.8.4^{\circ} \mathrm{E}\right)$. All three sites are beaches composed of an upper reflective beach face with a steep slope and a gently sloped dissipative sand flat in the lower intertidal. Both parts are usually separated by the bend (sudden change of slope angle), but the transition may become more gradual during periods without strong wave action, i.e. calm periods in summer or when the sea is covered by ice floes in winter. The beach 'Hausstrand' (sampled between $55.0154^{\circ} \mathrm{N}-55.0157^{\circ} \mathrm{N}$ and $8.4370^{\circ} \mathrm{E}-8.4390^{\circ} \mathrm{E}$ ) is the original site studied since 1965 and Schmidt [68] gave detailed descriptions on its morphology and physical factors. The two additional sites were both located in the southern part of the Königshafen tidal zone some $500 \mathrm{~m}$ north of Hausstrand (Fig. 1). 'Lister Haken' $\left(55.0243^{\circ} \mathrm{N}-55.0294^{\circ} \mathrm{N}\right.$ and $\left.8.4296^{\circ} \mathrm{E}-8.4351^{\circ} \mathrm{E}\right)$ is a

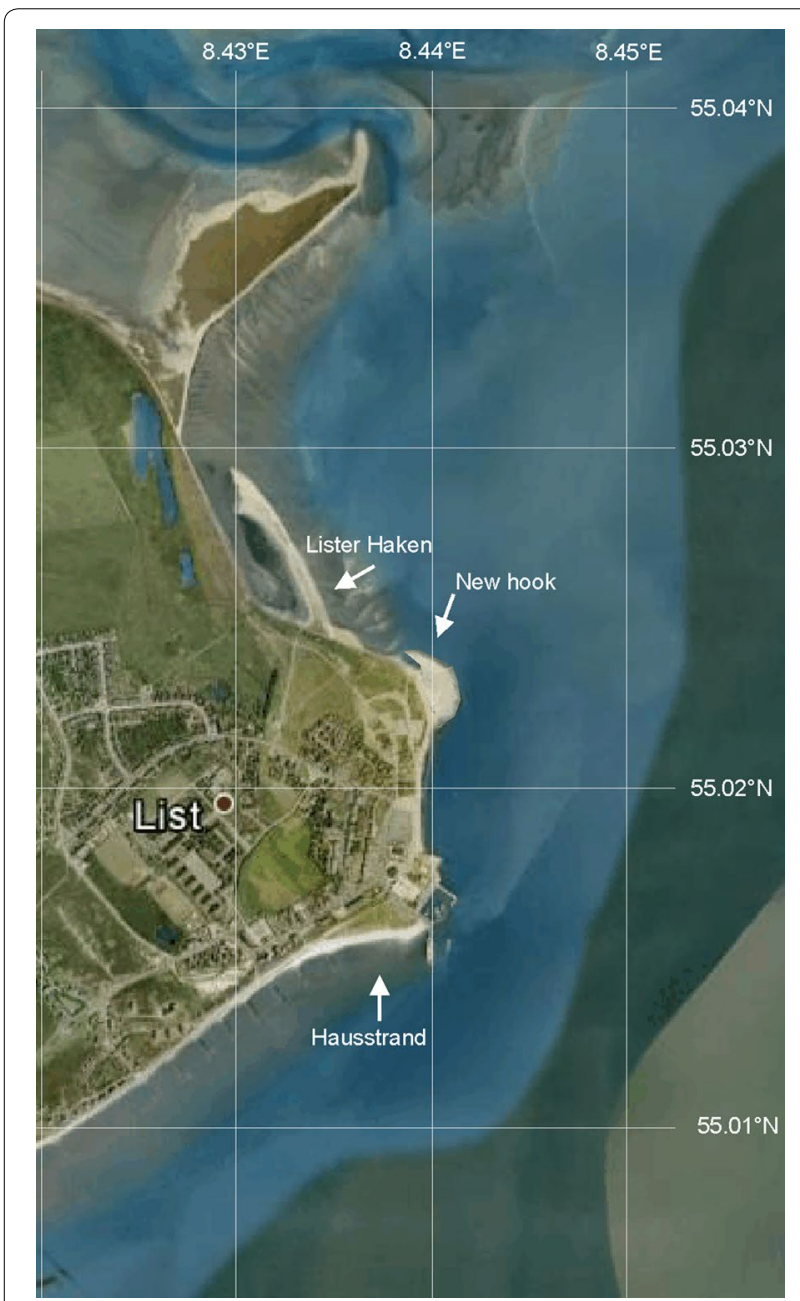

Fig. 1 Sampled sites near List, Island of Sylt, North Sea
$0.7 \mathrm{~km}$ long sandy spit with slightly coarser sediment than Hausstrand, while 'New hook' $\left(55.0233^{\circ} \mathrm{N}-55.0244^{\circ} \mathrm{N}\right.$ and $8.4374^{\circ} \mathrm{E}-8.4397^{\circ} \mathrm{E}$ ) has slightly finer sediment.

Sample collection generally followed the protocol proposed by Schmidt [68], i.e. sediment cores of $10 \mathrm{~cm}$ surface area reaching down well into the anoxic sediment layer and separated into $10 \mathrm{~cm}$-layers upon retrieval, but the number of cores per sampling date and location was less. In former times deeper sediment layers were sampled by digging holes into the beaches with a spade while I now used long Plexiglas pipes that were repeatedly driven into the same hole in the beach, each time retrieving a sediment layer of about $10 \mathrm{~cm}$. Below ground water level the sampling pipe was pushed down by partial vacuum. Depending on the position in the tidal gradient the sampled sediment depth varied between $5 \mathrm{~cm}$ close to low tide level and down to $100 \mathrm{~cm}$ at high tide level.

For faunal extraction the early studies all used the sea water ice method [80] as modified by Schmidt [68] which was the best method available at that time. Later extraction based on anaesthetization with $\mathrm{MgCl}_{2}$-solution proved to be superior, so the SMB-method [56] was used in this study. Field sampling commenced in March 2015 and lasted until July 2016, sampling all three beaches in random order but at least once a season. Thus 55 cores were retrieved from Hausstrand and 113 from Lister Haken and New hook (not separated because these areas share the lower intertidal zone), yielding a total of some 10,000 adult platyhelminth individuals (juveniles not included because they usually cannot be determined to species level). However, Acoelomorpha was excluded from this study because they are now placed apart from Platyhelminthes (e.g., [14] while this taxon was included 50 years ago.

In this study, abundance of all of the platyhelminth species was evaluated quantitatively for all of the cores. However, none of the previous studies contained comparable data because each author studied a single taxon only, and these studies are spread over a decade temporally. In addition, abundance data were commonly only given for single sampling dates and restricted to the most frequent species. But previous studies often contained information about the relative abundances of related taxa, so it was possible, at least, to identify the two or three most abundant species within a family (further on called 'dominants'). This ranking is compared to the ranking derived from the new data to find out whether or not the same species are numerical dominants today and 40-50 years ago.

\section{Results}

In the 1965-1975 period 168 platyhelminth species had been found in Hausstrand and 94 (56\%) of them were rediscovered during this study (Table 2). Including the two 
Table 2 Platyhelminth species recorded from Hausstrand between 1965 and 1975 and from 3 beaches in 2015 and 2016

\begin{tabular}{|c|c|c|c|c|c|}
\hline \multirow[t]{2}{*}{ Species } & \multirow{2}{*}{$\begin{array}{l}\text { 1965-1975 } \\
\text { Recorded by }\end{array}$} & \multicolumn{3}{|c|}{ 2015-2016 recorded in } & \multirow[t]{2}{*}{ Synonyms and remarks } \\
\hline & & Hausstrand & New hook & Lister Haken & \\
\hline \multicolumn{6}{|l|}{ Catenulida } \\
\hline Retronectes spec. & Faubel [28] & & 2 & & \\
\hline Rectronectes sterreri Faubel 1976 & Faubel [28] & 12 & 7 & & \\
\hline \multicolumn{6}{|l|}{ Macrostomida } \\
\hline Archimacrostomum pusillum (Ax, 1951) & Faubel [25] & & 34 & 281 & Macrostomump. \\
\hline Antromacrostomum armatum Faubel 1974 & Faubel [29] & & 157 & 5 & \\
\hline Bradynectes syltensis Faubel 1974 & Faubel [29] & 1 & 2 & & B. sterreri \\
\hline Bradynectes sterreri Rieger 1971 & - & & 2 & & Kristineberg form \\
\hline Microstomum jenseni Riedel 1932 & Faubel [29] & & 192 & & \\
\hline Microstomum papillosum Graff 1882 & Faubel [29] & 25 & & & \\
\hline Microstomum spiculifer Faubel 1974 & Faubel [29] & 1 & & & \\
\hline Myozona purpurea Faubel 1974 & Faubel [29] & 42 & 59 & 2 & \\
\hline Myozona stylifera Ax 1956 & Faubel [25] & & & & \\
\hline Paromalostomum dubium (Beauchamp 1927) & Pawlak [57] & 25 & 1 & 1 & \\
\hline Paromalostomum fusculum Ax 1952 & Pawlak [57] & 47 & 12 & 6 & \\
\hline Paromalostomum proceracauda Pawlak 1969 & Pawlak [57] & & & & \\
\hline \multicolumn{6}{|l|}{ Haplopharyngida } \\
\hline Haplopharynx rostratus Meixner 1938 & Faubel [25] & 7 & & 21 & \\
\hline \multicolumn{6}{|l|}{ Prolecithophora } \\
\hline Archimonotresis limophila Meixner 1938 & - & 1 & 7 & 2 & \\
\hline Pseudostomum quadrioculatum (Leuckart 1847) & - & 1 & 3 & & \\
\hline Pseudostomum gracilis Westblad 1955 & - & 18 & 21 & & \\
\hline \multicolumn{6}{|l|}{ Proseriata } \\
\hline \multicolumn{6}{|l|}{ Monocelididae } \\
\hline Archiloa petiti Ax 1956 & - & 1 & 5 & & \\
\hline Archilopsis arenaria Martens et al. 1989 & Sopott [73] & 16 & 11 & 25 & A. unipunctata \\
\hline Archilopsis marifuga Martens et al. 1989 & - & & 4 & 17 & A. unipunctata \\
\hline Archilopsis spinosa (Jensen 1878) & - & & & 4 & A. unipunctata \\
\hline Archilopsis unipunctata (Fabricius 1826) & - & & 12 & 13 & A. unipunctata \\
\hline Monocelopsis septentrionalis (Sopott 1972) & Sopott [73] & & 26 & 9 & Mesodas. \\
\hline Monocelis fusca Oersted 1843 & Sopott [73] & 1 & 1 & 23 & \\
\hline Monocelis lineata (Müller, 1773) & Sopott [73] & 1 & 4 & 70 & \\
\hline Promonotus marci Ax 1954 & Sopott [73] & 8 & 9 & & \\
\hline Promonotus schultzei Meixner 1943 & Sopott [73] & & & 21 & \\
\hline \multicolumn{6}{|l|}{ Archimonocelididae } \\
\hline $\begin{array}{l}\text { Archimonocelis crucifera Martens \& Curini-Galletti } \\
1993\end{array}$ & - & 4 & & & \\
\hline \multicolumn{6}{|l|}{ Coelogynoporidae } \\
\hline Carenscoilia bidentata Sopott 1972 & Sopott [73] & 6 & & & \\
\hline Carenscoilia biforamen Sopott 1972 & Sopott [73] & & & & \\
\hline Cirrifera aculeata (Ax 1951) & Sopott [73] & & & & \\
\hline Cirrifera boletiformae Sopott 1972 & Sopott [73] & 3 & & & \\
\hline Cirrifera cirrifera Sopott 1972 & Sopott [73] & & & 18 & \\
\hline Cirrifera dumosa Sopott 1972 & Sopott [73] & & 2 & & \\
\hline Coelogynopora axi Sopott 1972 & Sopott [73] & 1 & & & \\
\hline Coelogynopora biarmata Steinböck 1924 & Sopott [73] & & & & \\
\hline Coelogynopora distortofolio Sopott 1972 & Sopott [73] & & & 1 & \\
\hline Coelogynopora gynocotyla Steinböck 1924 & Sopott [73] & 2 & 6 & 2 & \\
\hline
\end{tabular}


Table 2 continued

\begin{tabular}{|c|c|c|c|c|c|}
\hline \multirow[t]{2}{*}{ Species } & \multirow{2}{*}{$\begin{array}{l}\text { 1965-1975 } \\
\text { Recorded by }\end{array}$} & \multicolumn{3}{|c|}{ 2015-2016 recorded in } & \multirow[t]{2}{*}{ Synonyms and remarks } \\
\hline & & Hausstrand & New hook & Lister Haken & \\
\hline Coelogynopora schulzii Meixner 1938 & Sopott [73] & & 1 & 2 & \\
\hline Coelogynopora steinboecki Sopott-Ehlers 1980 & - & 4 & & & \\
\hline Coelogynopora multispina n.sp. & - & & 1 & 1 & \\
\hline Coelogynopora solifer Sopott 1972 & Sopott [73] & & & 2 & \\
\hline Coelogynopora tenuis Meixner 1938 & Sopott [73] & & & & \\
\hline Stilivannuccia umbilica (Sopott, 1972) & Sopott [73] & & & & Vannuccia u. \\
\hline \multicolumn{6}{|l|}{ Otoplanidae } \\
\hline Bulbotoplana acephala Ax 1956 & Sopott [73] & 308 & 81 & 29 & \\
\hline Dicoelandropora atriopapillata Ax 1956 & Sopott [73] & 2 & & & \\
\hline Itaspiella helgolandica (Meixner 1938) & Sopott [73] & & & & \\
\hline Notocaryoplana arctica Steinböck 1935 & Sopott [73] & 10 & 1 & & Notocaryoplanella glandulosa \\
\hline Monostichoplana filum (Meixner 1938) & Sopott [73] & & & & Orthoplanaf. \\
\hline Otoplanella schulzi (Ax 1951) & Sopott [73] & 5 & 25 & 35 & \\
\hline Otoplanidia endocystis Meixner 1938 & Sopott [73] & & 9 & 5 & \\
\hline Parotoplana capitata Meixner 1938 & Sopott [73] & 2 & 1 & 12 & \\
\hline Parotoplana papii Ax 1956 & Sopott [73] & 1 & 278 & 25 & \\
\hline Parotoplanina geminoducta Ax 1956 & Sopott [73] & 38 & 2 & 68 & \\
\hline Philosyrtis rotundicephala Sopott 1972 & Sopott [73] & & & & \\
\hline Praebursoplana reisingeri Ax 1956 & - & 40 & 19 & 3 & \\
\hline Praebursoplana steinboecki Ax 1956 & Sopott [73] & 1 & & & \\
\hline \multicolumn{6}{|l|}{ Polystyliphoridae } \\
\hline Polystyliphora filum Ax 1958 & Sopott [73] & 16 & 2 & 9 & \\
\hline \multicolumn{6}{|l|}{ Nematoplanidae } \\
\hline Nematoplana coelogynoporoides Meixner 1938 & Sopott [73] & 818 & 143 & 390 & \\
\hline \multicolumn{6}{|l|}{ Dalytyphloplanida } \\
\hline \multicolumn{6}{|l|}{ Dalyelliidae } \\
\hline Halammovortex macropharynx (Meixner 1938) & - & 2 & & 2 & \\
\hline \multicolumn{6}{|l|}{ Graffillidae } \\
\hline Bresslauilla relicta Reisinger 1929 & Ehlers [20] & 10 & 13 & 4 & \\
\hline Orostylis falcatus n.n. & - & 37 & 3 & & "Bresslauilla aurora" \\
\hline Orostylis distortus n.n. & - & 7 & 8 & & "Bresslauilla Juni-Typ" \\
\hline Pseudograffilla arenicola Meixner 1938 & Ehlers [20] & & 5 & 24 & \\
\hline \multicolumn{6}{|l|}{ Provorticidae } \\
\hline Balgetia semicirculifera Karling 1962 & - & & & 11 & \\
\hline Baicalellia brevituba (Luther 1921) & - & & & 2 & \\
\hline Drepanilla limophila Ehlers 1979 & Ehlers [20] & 5 & & & \\
\hline Hangethellia calceifera Karling 1940 & - & 1 & 32 & 13 & \\
\hline Haplovejdovskya scilliensis Faubel \& Warwick 2005 & - & & 24 & & \\
\hline Pogaina kinnei Ax 1970 & Ehlers [20] & 38 & 14 & & \\
\hline Pogaina suecica (Luther 1948) & Ehlers [20] & 20 & 38 & 10 & \\
\hline Pogaina natans (Ax 1951) & - & 1 & & & \\
\hline Provortex balticus (Schultze 1851) & - & & 8 & 105 & \\
\hline Provortex psammophilus Ax 1951 & Ehlers [20] & 212 & 264 & 237 & \\
\hline Provortex tubiferus Luther 1948 & - & & 29 & & \\
\hline Nesite ptychoperoides n.g. n.sp. & - & & 7 & & \\
\hline \multicolumn{6}{|l|}{ Solenopharyngidae } \\
\hline Adenopharynx mitrabursalis Ehlers 1972 & Ehlers [20] & 23 & 10 & 16 & \\
\hline Anthopharynx vaginatus Karling 1940 & Ehlers [20] & 4 & 3 & 1 & A. sacculipenis \\
\hline
\end{tabular}


Table 2 continued

\begin{tabular}{|c|c|c|c|c|c|}
\hline \multirow[t]{2}{*}{ Species } & \multirow{2}{*}{$\begin{array}{l}\text { 1965-1975 } \\
\text { Recorded by }\end{array}$} & \multicolumn{3}{|c|}{$2015-2016$ recorded in } & \multirow[t]{2}{*}{ Synonyms and remarks } \\
\hline & & Hausstrand & New hook & Lister Haken & \\
\hline Aulopharynx aestuarius Ehlers 1972 & Ehlers [20] & 5 & 8 & & \\
\hline Doliopharynx geminocirro Ehlers 1972 & Ehlers [19] & & 2 & 3 & \\
\hline Proceropharynx anophthalmus (Meixner 1929) & Ehlers [19] & & 39 & & \\
\hline Proceropharynx litoralis Ehlers 1972 & Ehlers [20] & 30 & 16 & 7 & \\
\hline Tensopharynx inermis Ehlers 1972 & Ehlers [19] & & 3 & & \\
\hline Trisaccopharynx spiculatus Ehlers 1972 & Ehlers [19] & 2 & & & \\
\hline Solenopharyngid with 3 cirri & - & & 8 & & New species \\
\hline \multicolumn{6}{|l|}{ Typhloplanidae } \\
\hline Haloplanella hamulata Ehlers 1974 & Ehlers [21] & & 6 & 25 & \\
\hline Haloplanella longatuba Ax \& Heller 1970 & Ehlers [20] & 183 & & 11 & \\
\hline Haloplanella magnarhabdita n.sp. & - & 12 & & & \\
\hline Haloplanella minuta Luther 1946 & - & & & 1 & \\
\hline Haloplanella spiralis n.sp. & - & 4 & & & \\
\hline Hoplopera pusilla Ehlers 1974 & Ehlers [21] & & & & \\
\hline Pratoplana salsa Ax 1960 & - & & 3 & 26 & \\
\hline Pratoplana galeata Ehlers 1974 & Ehlers [20] & 178 & 59 & 134 & \\
\hline \multicolumn{6}{|l|}{ Promesostomidae } \\
\hline Adenorhynchus balticus Meixner 1938 & Ehlers [20] & 147 & 125 & 108 & \\
\hline Brinkmanniella macrostomoides Luther 1948 & Ehlers [20] & 52 & 6 & 22 & \\
\hline Brinkmanniella procerastyla Ehlers 1974 & Ehlers [21] & & & & \\
\hline Coronhelmis inaequalis n.sp. & - & 2 & 9 & & \\
\hline Coronhelmis inornatus Ehlers, 1974 & Ehlers [21] & & & & \\
\hline Coronhelmis lutheri Ax, 1951 & Ehlers [21] & & & & \\
\hline Coronhelmis multispinosus Luther, 1948 & Ehlers [21] & 4 & & & \\
\hline Coronhelmis tripartitus Ehlers, 1974 & Ehlers [21] & 2 & 2 & & \\
\hline Listea simplex Ax \& Heller 1970 & Ehlers [20] & 23 & & 9 & \\
\hline Litucivis serpens Ax \& Heller 1970 & Ehlers [20] & 138 & 4 & 4 & \\
\hline Moevenbergia oculofagi n.sp. & - & 1 & 6 & & \\
\hline Promesostoma bipartitum Ax 1956 & Ehlers [21] & & & & \\
\hline Promesostoma caligulatum Ax 1952 & Ehlers [20] & & 6 & 4 & \\
\hline Promesostoma cochleare Karling 1935 & - & & & 1 & \\
\hline Promesostoma gracilis Ax 1951 & Ehlers [20] & 3 & 7 & & \\
\hline Promesostoma karlingi Ehlers 1974 & Ehlers [21] & & 20 & & \\
\hline Promesostoma marmoratum (M.Schultze 1851) & Ehlers [20] & 1 & 20 & 1 & \\
\hline Promesostoma meixneri Ax 1951 & Ehlers [20] & 14 & 49 & 2 & \\
\hline Promesostoma minutum Ax 1956 & - & 3 & 8 & & \\
\hline Promesostoma rostratum Ax, 1951 & Ehlers [20] & & 11 & & \\
\hline Promesostoma serpentistylum Ax 1952 & Ehlers [20] & 51 & 4 & 34 & \\
\hline Protandrella cirrifera Ehlers et al. 1994 & Ehlers et al. [22] & & & & \\
\hline Scoliopharyngia arenicola Ehlers et al. 1995 & - & & 2 & & \\
\hline Scoliopharyngia listensis Ehlers et al. 1994 & Ehlers et al. [22] & & & & \\
\hline Subulagera mucronata Ehlers 1974 & Ehlers [20] & 3 & & & \\
\hline Tvaerminnea direceptacula Ehlers et al. 1994 & Ehlers et al. [22] & 15 & & & \\
\hline Tvaerminnea karlingi Luther 1943 & - & 2 & & 2 & \\
\hline \multicolumn{6}{|l|}{ Ciliopharyngiellidae } \\
\hline Ciliopharyngiella intermedia Ax 1952 & Ehlers [20] & 47 & 18 & 29 & \\
\hline \multicolumn{6}{|l|}{ Trigonostomidae } \\
\hline Lonchoplanella axi Ehlers 1974 & Ehlers [20] & 203 & 6 & 1 & \\
\hline
\end{tabular}


Table 2 continued

\begin{tabular}{|c|c|c|c|c|c|}
\hline \multirow[t]{2}{*}{ Species } & \multirow{2}{*}{$\begin{array}{l}\text { 1965-1975 } \\
\text { Recorded by }\end{array}$} & \multicolumn{3}{|c|}{ 2015-2016 recorded in } & \multirow[t]{2}{*}{ Synonyms and remarks } \\
\hline & & Hausstrand & New hook & Lister Haken & \\
\hline Mariplanella frisia Ax \& Heller 1970 & Ehlers [20] & 1 & 47 & 44 & \\
\hline Messoplana elegans (Luther 1948) & - & & 1 & & \\
\hline Messoplana falcata (Ax 1953) & Ehlers [20] & 10 & 1 & 3 & \\
\hline Messoplana floralis Ehlers 1974 & Ehlers [21] & & & & \\
\hline Messoplana helgolandica Ax 1971 & - & 1 & & 4 & \\
\hline Messoplana rugata Ehlers 1974 & Ehlers [21] & & & 1 & \\
\hline Petaliella spiracauda Ehlers 1974 & Ehlers [20] & 40 & 219 & & \\
\hline Ptychopera ehlersi Ax 1971 & $A x[6]$ & & & & \\
\hline Ptychopera westbladi (Luther 1943) & - & & & 181 & \\
\hline Proxenetes ampullatus Ax 1971 & Ehlers [21] & & & & \\
\hline Proxenetes cimbricus Ax 1971 & - & 10 & 12 & 12 & \\
\hline Proxenetes deltoides Hartog 1965 & - & & & 41 & \\
\hline Proxenetes fasciger Ehlers 1974 & - & & 8 & & \\
\hline Proxenetes flabellifer Jensen 1878 & $A x[6]$ & & & & \\
\hline Proxenetes inflatus den Hartog 1966 & - & & 1 & & \\
\hline Proxenetes quadrispinosus den Hartog 1966 & $A x[6]$ & 3 & 15 & & \\
\hline Proxenetes quinquespinosus Ax 1971 & Ehlers [21] & & 2 & 1 & \\
\hline Proxenetes simplex Luther 1948 & $A x[6]$ & & & 3 & \\
\hline Proxenetes trigonus Ax 1960 & $A x[6]$ & & 13 & & \\
\hline \multicolumn{6}{|l|}{ Kalyptorhynchia } \\
\hline \multicolumn{6}{|l|}{ Cicerinidae } \\
\hline Cicerina brevicirrus Meixner 1928 & Schilke [67] & & 65 & 1 & \\
\hline Cicerina remanei Meixner 1928 & Hoxhold [39] & & 15 & 3 & \\
\hline Cicerina tetradactyla Giard 1904 & Hoxhold [39] & & 10 & & \\
\hline Paracicerina laboeica Meixner 1928 & Hoxhold [39] & 8 & & & \\
\hline Ptyalorhynchus coecus Ax 1951 & Hoxhold [39] & 31 & 21 & 4 & \\
\hline Zonorhynchus seminascatus Karling 1956 & Schilke [67] & & 11 & 7 & \\
\hline \multicolumn{6}{|l|}{ Cystiplanidae } \\
\hline Cystiplana karlingi Hoxhold n.n. & Hoxhold [39] & 1 & & & \\
\hline Cystiplana paradoxa Karling 1964 & Hoxhold [39] & 581 & 5 & 177 & \\
\hline Nigerrhynchus opisthoporus Schilke 1970 & Hoxhold [39] & 16 & 8 & & \\
\hline \multicolumn{6}{|l|}{ Gnathorhynchidae } \\
\hline Aculeorhynchus glandulis Schilke 1969 & Hoxhold [39] & 1 & & & \\
\hline Gnathorhynchus conocaudatus Meixner 1929 & Hoxhold [39] & 14 & 6 & & \\
\hline Gnathorhynchus inermis Schilke 1970 & Hoxhold [39] & & & 1 & \\
\hline Pterognathus longostilo (Schilke 1970) & Hoxhold [39] & & & & Neognathorhynchus longostilo \\
\hline Neognathorhynchus suecicus Karling 1956 & Hoxhold [39] & & 12 & & \\
\hline Odontorhynchus lonchiferus Karling 1947 & - & 1 & & & \\
\hline Paragnathorhynchus subterraneus Meixner 1938 & Hoxhold [39] & & & 1 & Prognathorhynchus stilofer \\
\hline Psittacorhynchus verweyi Hartog 1968 & - & & & 1 & \\
\hline Uncinorhynchus flavidus Karling 1947 & Schilke [67] & & & & \\
\hline \multicolumn{6}{|l|}{ Koinocystidae } \\
\hline Harenorhynchus proterandrius Hoxhold n.n. & Hoxhold [39] & & & & \\
\hline Parautelga bilioi Karling 1964 & Schilke [67] & & & & \\
\hline Marirhynchus longasaeta Schilke 1970 & Hoxhold [39] & 8 & & 29 & \\
\hline \multicolumn{6}{|l|}{ Placorhynchidae } \\
\hline Placorhynchus dimorphis Karling 1947 & Hoxhold [39] & & & 4 & P. octaculeatus d. \\
\hline Placorhynchus octaculeatus Karling 1931 & Schilke [67] & & 2 & 5 & \\
\hline
\end{tabular}


Table 2 continued

\begin{tabular}{|c|c|c|c|c|c|}
\hline \multirow[t]{2}{*}{ Species } & \multirow{2}{*}{$\begin{array}{l}\text { 1965-1975 } \\
\text { Recorded by }\end{array}$} & \multicolumn{3}{|c|}{ 2015-2016 recorded in } & \multirow[t]{2}{*}{ Synonyms and remarks } \\
\hline & & Hausstrand & New hook & Lister Haken & \\
\hline \multicolumn{6}{|l|}{ Polycystididae } \\
\hline Acrorhynchides robustus (Karling 1931) & Schilke [67] & 4 & 12 & 5 & \\
\hline Danorhynchus duplostylis Karling 1955 & Hoxhold [39] & & 1 & & \\
\hline Gyratrix hermaphroditus Ehrenberg 1831 & Hoxhold [39] & 78 & 4 & & \\
\hline Limipolycystis curvitubo Schilke 1970 & Hoxhold [39] & 4 & & & \\
\hline Neopolycystis tridentata Karling 1955 & Hoxhold [39] & 45 & 22 & 81 & \\
\hline Phonorhynchus bitubatus Meixner 1938 & - & & 1 & & \\
\hline Scanorhynchus forcipatus Karling 1955 & Hoxhold [39] & 1 & 15 & 1 & \\
\hline Typhlopolycystis coeca Karling 1956 & Hoxhold [39] & & & & \\
\hline Typhlopolycystis mediterranea Brunet 1965 & Hoxhold [39] & & & & T. limicola \\
\hline Typhlopolycystis indet. & - & 1 & & & New species \\
\hline Polycystididae indet. & - & 1 & & & New species \\
\hline Eukalyptorhynchia indet. & - & & 4 & & New species \\
\hline \multicolumn{6}{|l|}{ Psammorhynchidae } \\
\hline Psammorhynchus tubulipenis Meixner 1938 & Hoxhold [39] & 46 & 72 & 47 & \\
\hline \multicolumn{6}{|l|}{ Diascorhynchidae } \\
\hline Diascorhynchides arenaria Schilke 1970 & Hoxhold [39] & 14 & & & \\
\hline Diascorhynchus rubrus Boaden 1963 & Hoxhold [39] & 1 & 1 & & \\
\hline Diascorhynchus serpens Karling 1949 & Hoxhold [39] & & 1 & & \\
\hline Diascorhynchus falconis n. sp. & - & & 2 & & \\
\hline \multicolumn{6}{|l|}{ Nematorhynchidae } \\
\hline Nematorhynchus parvoacumine Schilke 1969 & Hoxhold [39] & & & & \\
\hline \multicolumn{6}{|l|}{ Schizorhynchidae } \\
\hline Amphirhynchus caudatus Schilke 1970 & Hoxhold [39] & 4 & & & \\
\hline Carcharodorhynchus ambronensis Schilke 1970 & Hoxhold [39] & & & & \\
\hline Carcharodorhynchus isolatus Schilke 1970 & Hoxhold [39] & & & & \\
\hline Carcharodorhynchus listensis Schilke 1970 & Hoxhold [39] & 28 & 57 & 13 & \\
\hline Carcharodorhynchus subterraneus Ax 1951 & Schilke [67] & & & & \\
\hline Neoschizorhynchus brevipharynggus Schilke 1970 & Hoxhold [39] & & 1 & & \\
\hline Neoschizorhynchus longipharynggus Schilke 1970 & Hoxhold [39] & & & & \\
\hline Neoschizorhynchus parvorostro Ax \& Heller 1970 & Hoxhold [39] & 2 & 48 & 1 & \\
\hline Paraschizorhynchoides glandulis Schilke 1970 & Hoxhold [39] & 3 & & & \\
\hline Proschizorhynchella bivaginata (Schilke 1970) & Hoxhold [39] & 2 & 4 & 2 & Proschizorhynchus $b$. \\
\hline Proschizorhynchella helgolandica (L'Hardy 1965) & Hoxhold [39] & 1 & 2 & & Proschizorhynchush. \\
\hline Proschizorhynchella robusta Noldt 1989 & - & & & 1 & \\
\hline Proschizorhynchella spiracirro (Schilke 1970) & Hoxhold [39] & 2 & 1 & & Proschizorhynchuss. \\
\hline Proschizorhynchus gullmarensis Karling 1950 & Hoxhold [39] & 126 & 32 & 276 & \\
\hline Proschizorhynchus triductibus Schilke 1970 & Hoxhold [39] & 8 & 13 & 3 & \\
\hline Pseudoschizorhynchoides ruber Schilke 1970 & Hoxhold [39] & & & & \\
\hline Schizochilus caecus L'Hardy 1963 & Hoxhold [39] & 1 & & & \\
\hline Schizochilus choriurus Boaden 1963 & Hoxhold [39] & 118 & 17 & 3 & \\
\hline Schizochilus marcusi Boaden 1963 & Hoxhold [39] & & & & \\
\hline Schizorhynchoides aculeatus L'Hardy 1963 & Hoxhold [39] & 14 & 80 & 34 & \\
\hline Schizorhynchoides brevistili Noldt 1989 & - & & 16 & & \\
\hline Schizorhynchoides canaliculatus L'Hardy 1963 & Hoxhold [39] & 1 & & & \\
\hline Schizorhynchoides rarus Ax 1951 & Hoxhold [39] & & & & \\
\hline Thylacorhynchus ambronensis Schilke 1970 & Schilke [67] & 1 & 3 & & \\
\hline Thylacorhynchus caudatus Meixner 1928 & Schilke [67] & 2 & & 2 & \\
\hline
\end{tabular}


Table 2 continued

\begin{tabular}{|c|c|c|c|c|c|}
\hline \multirow[t]{2}{*}{ Species } & \multirow{2}{*}{$\begin{array}{l}\text { 1965-1975 } \\
\text { Recorded by }\end{array}$} & \multicolumn{3}{|c|}{ 2015-2016 recorded in } & \multirow[t]{2}{*}{ Synonyms and remarks } \\
\hline & & Hausstrand & New hook & Lister Haken & \\
\hline Thylacorhynchus conglobatus Meixner 1928 & Hoxhold [39] & & 7 & & \\
\hline Thylacorhynchus longicirrus Noldt 1989 & - & 3 & & & \\
\hline Thylacorhynchus pyriferus Karling 1950 & Schilke [67] & & & 1 & \\
\hline \multicolumn{6}{|l|}{ Karkinorhynchidae } \\
\hline Baltoplana magna Karling 1949 & Hoxhold [39] & 1 & & & \\
\hline Cheliplana boadeni Schilke 1970 & Hoxhold [39] & 8 & 2 & 4 & \\
\hline Cheliplana gemmifera Noldt 1989 & - & & 6 & & \\
\hline Cheliplana remanei (Meixner 1928) & Hoxhold [39] & 75 & 16 & 5 & \\
\hline Cheliplana vestibularis Beauchamp 1927 & - & & 39 & & C. curvocirra \\
\hline Cheliplanilla caudata Meixner 1938 & Hoxhold [39] & & 48 & 1 & \\
\hline Karkinorhynchus bruneti Schilke 1970 & Hoxhold [39] & 1 & 6 & & \\
\hline Karkinorhynchus lehardyi Schilke 1970 & Hoxhold [39] & & & & \\
\hline Karkinorhynchus listensis Schilke 1970 & Hoxhold [39] & & & & \\
\hline Karkinorhynchus primitivus Meixner 1928 & - & 2 & & 1 & \\
\hline Karkinorhynchides purpureus (Schilke 1970) & Hoxhold [39] & & 9 & 1 & Karkinorhynchus p. \\
\hline
\end{tabular}

-, no published record; in case of multiple records, only the first record is given. Ciphers in 2015/16 records give the number of adults determined to species level

adjoining beaches the number of re-discovered species increases to 131 (78\%). Among the numerical dominant species the rate of re-discovery was even higher and most of the numerical dominants of the 1965-1975 era were still among the dominants in the present study, indicating a high temporal stability of species composition (Table 3). In 19 out of 23 families the same species claimed the top ranks of abundance though their order changed in half of the families. Remarkable exceptions were Antromacrostomum armatum and Microstomum jenseni with previous top ranks of abundance in their families (Table 3) while both species were not recorded at all in the present study in Hausstrand but reached top abundances in New hook (Table 2). In Proseriata the otoplanid Itaspiella helgolandica was no longer recorded in 2015-2016 while the parotoplanid Praebursoplana reisingeri took the top rank in 2015-2016 but had not been recorded previously (Table 3).

The 2015/16 Hausstrand samples gave a total of 119 species and 94 (79\%) of them had already recorded there previously. Thus, 25 species had not been found before in Hausstrand (Table 2), but 15 of them were previously known from other habitats on the island of Sylt. The two adjoining beaches also harbored species that had been unknown from Hausstrand but often known from other habitats. Nevertheless, 20 out of the total 220 platyhelminth species found during this study were not previously reported from the island of Sylt (Table 4).

These 20 'first records' include 13 undescribed species, but unpublished records indicate that at least 3 of them had already been present in the Sylt area some decades ago (Table 4). Among the new species described in this paper all but Nesite ptychoperoides n. sp. belong to genera that are well established in the eastern Atlantic. For 4 species the developmental stage of the individuals found was insufficient for a formal description. Finally, 7 of the 20 'new' species have already been described from elsewhere but are first records for the island of Sylt potentially including invasive or introduced species:

Bradynectes sterreri was reported from Hausstrand by Faubel $[25,26]$, but later regarded a species of its own: B. syltensis Faubel \& Warwick, 2005. Whether or not $B$. sterreri in the strict sense (i.e. the 'Kristineberg form' in Rieger [65] already occurred in the 1960s is not clear.

Pseudostomum gracilis was never reported from the island of Sylt, however, the taxon Prolecithophora was rarely studied and not included in the previous Hausstrand studies. So far, the species is only known from the Gullmarsfjord in Sweden [82].

Archimonocelis crucifera was described from the Mediterranean [44] and has not been reported outside the Mediterranean. However, most species of Archimonocelis occur in the subtidal which has poorly been studied in the Sylt area. Nevertheless, a recent invasion of this species cannot be excluded.

Haplovejdovskya scilliensis was described from the Island of Scilly but unpublished records indicate it occurred on the Island of Sylt for 40 years, at least, and is not a recent invader.

Promesostoma minutum occurs along the French Atlantic coast [7] and was never reported for the Island of Sylt. However, it is very similar to $P$. meixneri but lacks the bursal appendage typical for the latter species. 
Table 3 Numerical dominant platyhelminth species in abundant families in Hausstrand 1965-1975 (according to source of ranking) and 2015-2016 (according to Table 2)

\begin{tabular}{|c|c|c|c|c|c|}
\hline \multirow[t]{2}{*}{ Taxon } & \multirow[t]{2}{*}{ Family } & \multirow[t]{2}{*}{ Species } & \multicolumn{2}{|c|}{ Rank of abundance } & \multirow[t]{2}{*}{ Source of ranking } \\
\hline & & & 1965-1975 & 2015-2016 & \\
\hline \multicolumn{6}{|c|}{ Macrostomida } \\
\hline & Haplopharyngidae & Haplopharynx rostratus & 1 & 1 & Faubel $[25,26$, p. 29] \\
\hline & Dolichomacrostomidae & Paromalostomum fusculum & 1 & 1 & Pawlak [57, p. 443] \\
\hline & & Paromalostomum dubium & 2 & 2 & Pawlak [57, p. 445] \\
\hline & Macrostomidae & Antromacrostomum armatum & 1 & - & Faubel $[29$, p. 26] \\
\hline & & Myozona purpurea & 2 & 1 & Faubel [29, p. 27] \\
\hline & Microstomidae & Microstomum jenseni & 1 & - & Faubel $[29$, p. 66] \\
\hline & & Microsomum papillosum & 2.5 & 1 & Faubel $[29$, p. 48] \\
\hline & & Microstomum spiculifer & 2.5 & 2 & Faubel $[29$, p. 49] \\
\hline \multicolumn{6}{|c|}{ Proseriata } \\
\hline & Bulbotoplaninae & Bulbotoplana acephala & 1 & 1 & Sopott $[74$, p. 48$]$ \\
\hline & Otoplaninae & Itaspiella helgolandica & 1.5 & - & Sopott $[73$, p. 58] \\
\hline & & Otoplanella schulzi & 1.5 & 2 & Sopott $[73$, p. 59] \\
\hline & & Notocaryoplana arctica & 3 & 1 & Sopott $[73$, p. 60] \\
\hline & Parotoplaninae & Parotoplana capitata & 1.5 & 3 & Sopott $[73$, p. 50] \\
\hline & & Parotroplanina geminoducta & 1.5 & 2 & Sopott $[73$, p. 50] \\
\hline & & Parotoplanapapii & 3.5 & 4 & Sopott $[73$, p. 51] \\
\hline & & Praebursoplana reisingeri & - & 1 & \\
\hline & Nematoplanidae & Nematoplana coelogynoporoides & 1 & 1 & Sopott $[73$, p. 62] \\
\hline \multicolumn{6}{|c|}{ Dalytyphloplanida } \\
\hline & Provorticidae & Provortex psammophilus & 1 & 1 & Ehlers $[20$, p. 61] \\
\hline & & Pogaina suecica & 2.5 & 3 & Ehlers $[20$, p. 61] \\
\hline & & Pogaina kinnei & 2.5 & 2 & Ehlers $[20$, p. 61] \\
\hline & Solenopharyngidae & Proceropharynx litoralis & 1 & 1 & Ehlers $[20$, p. 30] \\
\hline & Adenorhynchinae & Litucivis serpens & 1.5 & 2 & Ehlers $[20$, p. 34] \\
\hline & & Adenorhynchus balticus & 1.5 & 1 & Ehlers [20, p. 34] \\
\hline & & Listea simplex & 3 & 3 & Ehlers $[20$, p. 34] \\
\hline & Promesostominae & Promesostoma meixneri & 1 & 2 & Ehlers $[20$, p. 34] \\
\hline & Typhloplanidae & Haloplanella longatuba & 1.5 & 1.5 & Ehlers $[20$, p. 20] \\
\hline & & Pratoplana galeata & 1.5 & 1.5 & Ehlers [20, p. 20] \\
\hline & Trigonostomidae & Lonchoplanella axi & 2 & 1 & Ehlers $[20$, p. 51] \\
\hline & & Mariplanella frisia & 2 & 4 & Ehlers $[20$, p. 51] \\
\hline & & Petaliella spiracauda & 2 & 2 & Ehlers [20, p. 51] \\
\hline & & Messoplana falcata & 4 & 3 & Ehlers [21, p. 72] \\
\hline \multicolumn{6}{|c|}{ Kalyptorhynchia } \\
\hline & Cicerinidae & Ptyalorhynchus coecus & 2 & 1 & Hoxhold [39, p. 74] \\
\hline & & Paracicerina laboeica & 1 & 2 & Hoxhold [39, p. 112] \\
\hline & Cystiplanidae & Cystiplana paradoxa & 1 & 1 & Hoxhold [39, p. 118] \\
\hline & & Cystiplana karlinginn & 2 & 3 & Hoxhold [39, p. 59] \\
\hline & & Nigerrhynchus opisthoporus & 3 & 2 & Hoxhold [39, p. 93] \\
\hline & Gnathorhynchidae & Gnathorhynchus conocaudatus & 1 & 1 & Hoxhold [39, p. 112] \\
\hline & Koinocystididae & Marirhynchus longasaeta & 1 & 1 & Hoxhold [39, p. 114] \\
\hline & Polycystididae & Gyratrix hermaphroditus & 1 & 1 & Hoxhold [39, p. 127] \\
\hline & & Neopolycystis tridentata & 2 & 2 & Hoxhold [39, p. 49] \\
\hline & Cheliplanidae & Cheliplana boadeni & 1 & 2 & Hoxhold [39, p. 126] \\
\hline & & Cheliplana remanei & 2 & 1 & Hoxhold [39, p. 27] \\
\hline & Diascorhynchidae & Diascorhynchides arenaria & 1 & 1 & Hoxhold [39, p. 112] \\
\hline
\end{tabular}


Table 3 continued

\begin{tabular}{|c|c|c|c|c|c|}
\hline \multirow[t]{2}{*}{ Taxon } & \multirow[t]{2}{*}{ Family } & \multirow[t]{2}{*}{ Species } & \multicolumn{2}{|c|}{ Rank of abundance } & \multirow[t]{2}{*}{ Source of ranking } \\
\hline & & & $1965-1975$ & 2015-2016 & \\
\hline & Karkinorhynchidae & Karkinorhynchus lehardyi & 1 & 3 & Hoxhold [39, p. 112] \\
\hline & Schizorhynchidae & Schizochilus choriurus & 1 & 2 & Hoxhold [39, p. 123] \\
\hline & & Proschizorhynchus gullmarensis & 2 & 1 & Hoxhold [39, p. 119] \\
\hline & & Schizochilus caecus & 3 & 7 & Hoxhold $[39$, p. 121] \\
\hline & & Schizorhynchoides aculeatus & 4 & 4 & Hoxhold [39, p. 115] \\
\hline & & Paraschizorhynchoides glandulis & 6 & 6 & Hoxhold [39, p. 114] \\
\hline & & Carcharodorhynchus listensis & 5 & 3 & Hoxhold [39, p. 124] \\
\hline & & Proschizorhynchus triductibus & 7 & 5 & Hoxhold [39, p. 87] \\
\hline
\end{tabular}

Identical ranks within families refer to identical abundance information in the sources. -, not recorded

Table 4 Platyhelminth species in the study sites not previously recorded from the island of Sylt

\begin{tabular}{|c|c|c|c|c|}
\hline \multirow[t]{2}{*}{ Species } & \multicolumn{3}{|c|}{ 2015-2016 recorded in } & \multirow[t]{2}{*}{ Remarks } \\
\hline & Hausstrand & New hook & Lister Haken & \\
\hline \multicolumn{5}{|l|}{ First records } \\
\hline Bradynectes sterreri Rieger 1971 & & 2 & & Kristineberg form \\
\hline Pseudostomum gracilis Westblad 1955 & 18 & 21 & & \\
\hline Archimonocelis crucifera Martens \& Curini-Galletti 1993 & 4 & & & \\
\hline Haplovejdovskya scilliensis Faubel \& Warwick 2005 & & 24 & & \\
\hline Promesostoma minutum Ax 1956 & 3 & 8 & & \\
\hline Messoplana helgolandica Ax 1971 & 1 & & 4 & \\
\hline Proxenetes inflatus den Hartog 1966 & & 1 & & \\
\hline \multicolumn{5}{|l|}{ New species that can be described } \\
\hline Coelogynopora multispina n. sp. & & 1 & 1 & \\
\hline Orostylis falcatus nom. nud. ${ }^{a}$ & 41 & 3 & & Unpublished records \\
\hline Orostylis distortus nom. nud. ${ }^{a}$ & 8 & 8 & & Unpublished records \\
\hline Nesite ptychoperoides n. g., n. sp. & & 7 & & \\
\hline Haloplanella magnarhabdita n. sp. & 12 & & & \\
\hline Haloplanella spiralis n. sp. & 4 & & & \\
\hline Coronhelmis inaequalis n. sp. & 2 & 9 & & \\
\hline Moevenbergia oculofagi n. sp. & 1 & 6 & & Unpublished records \\
\hline Diascorhynchus falconis n. sp. & & 2 & & \\
\hline \multicolumn{5}{|l|}{ New species, insufficient for a formal description } \\
\hline Solenopharyngid with 3 cirri & & 8 & & \\
\hline Typhlopolycystis sp. & 1 & & & \\
\hline Polycystididae indet. & 1 & & & \\
\hline Eukalyptorhynchia indet. & & 4 & & \\
\hline
\end{tabular}

Figures give the number of specimens found

a Described separately

Unpublished files of a 'P. meixneri without bursal appendage' indicate that it may have been present on the Island of Sylt but confused with not fully-developed individuals of P. meixneri.

Messoplana helgolandica was described from the island of Helgoland [6] while the genus is represented on the Island of Sylt by the closely related M. floralis Ehlers
1974. However, the separation of these species is not very convincing because both discriminating characters (shape and degree of hardening of the proximal bursal appendage and mouthpiece of the seminal receptacle with or without a small hardened ring) given by Ehlers [21] depend on the developmental state of the specimens and are hardly seen in smaller individuals. 
Finally, Proxenetes inflatus was only known from a single locality at the Swedish west coast [33] and had never been re-discovered before this study.

Thus, among the species recorded for the first time there is no convincing evidence for a recent invasion.

\section{Discussion}

An unambiguous identification of species as invaders or introduced species depends on our knowledge of their original biogeographic ranges. This knowledge tends to increase with body size and/or economic importance of the species. Accordingly, our knowledge on meiofauna is limited and it is particularly scant in the soft-bodied taxa such as platyhelminths that are best studied alive and therefore rarely included in faunal surveys. Often the 'natural distributional range' of a platyhelminth species is merely a freehanded circle around few localities with 'true' range limits unknown. On a world wide scale, the number of observations is highest in Europe with obvious hotspots around the marine biological stations. Thus, changes in the geographical distribution of platyhelminth species can only be detected at these well studied sites and the sudden occurrence of species formerly unknown from the respective area is the only hint for a change.

However, a first record of a species in a given study site may have multiple reasons. The first is hidden diversity resulting from patchiness, i.e. small-scale spatial inhomogeneity in distribution pattern, potentially combined with high short-term temporal variability (e.g., [77]. For abundant species this means problems in reliable quantification while species with narrow spatial and/or temporal niches have a significant chance to remain undetected at all, even in highly-replicated sampling designs. This may be the main reason why some of the species known from previous studies are not recorded in this study. On the other hand, the 25 species new for Hausstrand during this study indicate that thousands of sediment cores studied in previous times were not enough to reach a saturation level in species/area curves. So far, we do not know the full set of species from any locality, some species always remain undetected in meiofauna studies. Hyper-cryptic species (i.e. species clearly separated genetically but showing little or no morphological differences) add to the complexity (e.g., [15].

Temporal change of the physical properties of a site may be another cause for changes in species composition because any physical change may favor some of the species, enabling higher abundance, and deteriorate the living conditions and abundance for other species. Since our chances to record these species vary with abundance, we are likely to record differences in species composition merely for statistical reasons. True change in species composition may occur if the changes in physical properties enable species from neighboring sites to immigrate while other species possibly emigrate or go locally extinct. The changes in species composition among the numerical dominants (Table 3) may be an example for that. The chance to identify such 'exchange of species between neighboring habitats' depends on the number of physically different sites studied within an area. For the Island of Sylt our knowledge on sites that are physically similar, but not identical to Hausstrand, was high enough to identify 15 out of 25 species recorded for the first time in Hausstrand to be either immigrants from neighboring sites, or winners of physical variations and therefore first recorded due to of higher abundance (or, possibly, by mere chance). In addition, the higher efficiency of the $\mathrm{MgCl}_{2}$ extraction method may have increased the rate of recorded species.

The species that are new to science of course have no known geographic distribution but the type locality. Therefore the question of their origin (resident, immigrant or introduced) cannot be answered until more records become available. For new species that classify into known genera we can only check whether or not the new species fits the geographic distribution of the known ones. A new species outside the known range of a genus may be reasonably suspected as a case of species introduction. But again we face the problem of limited knowledge on the geographic range, now on the level of genera. By now, many genera seem to be confined to single oceanic basins such as the Mediterranean or the North Atlantic, but in many cases this may be an artifact of limited knowledge on the platyhelminth fauna on a global scale. Or, as pointed out by Artois et al. [4], distribution maps of species of microturbellaria actually reflect the distributions of taxonomists, and the expeditions they have done. As an example, the number of published records on meiofaunal platyhelminths all along the Atlantic continental coastline of Africa is zero. On a world wide scale the total number of free living marine platyhelminth species is estimated to be 3-12 times higher than the number described by know [1]. Accordingly, any conclusions on change in local platyhelminth species composition are preliminary estimates strongly hampered by huge geographic gaps of knowledge.

In other meiofaunal taxa the geographic gaps of knowledge may be smaller but the number of immigrated or introduced species recorded is also very low (e.g., [24, 38]. This coincides with the rarity of pelagic larvae because in most meiofaunal groups juveniles develop within the sedimentary habitat, and their number is generally low. Therefore Sterrer [76] concluded that meiofauna might generally exhibit a very low rate of dispersal. Analyzing the meiofaunal means of dispersal in more detail, Gerlach [32] concluded that benthic animals with pelagic larvae have a better year-to-year chance than meiofauna to establish populations in other areas, while meiofauna 
without a restricted larval period may be superior in using storms of various grades for long distance dispersal. Thus, there may be no general difference in the dispersal ability of meio- and macrobenthos in the long run but possibly a higher temporal variability in the meiofaunal chances to disperse. Concerning man-induced transport, meio- and macrobenthic species use the same vectors, i.e. ships ballast water tanks [59], epigrowth on the hull [30], and material transported for aquaculture [72]. Thus, the scantiness of records on non-native meiofaunal species is not a meiofaunal feature but most probably an artifact of a lack of studies. The following species descriptions aim to fill a bit of our knowledge gaps.

\section{Species descriptions}

With the intense studies on platyhelminth species in previous years (Table 1), the locally abundant species seem to be known by now, and only species with a low local abundance are left undescribed. Accordingly, the number of individuals that could be studied was very low for the new species. Most of this material was used for live observation in squeezing preparation which usually means the animals are left damaged after the observations and are no longer suitable for permanent slides. Corresponding to article 73.1.4 of the International Code of Zoological Nomenclature (Fourth Edition) I therefore will designate the illustrations of the holotype specimens recorded during the microscopic study as the holotype.

\section{Coelogynopora multispina n. sp. (Figs. 2, 3)}

Localities (1) Island of Sylt, Lister Haken, sheltered western beach face (locus typicus, $55.0248^{\circ} \mathrm{N}, 8.4333^{\circ} \mathrm{E}$ ). Medium to coarse sand $0.3 \mathrm{~m}$ below mean high tide level, 15-20 cm below the surface (March 2015, 1 individual). (2) Island of Sylt, semi-exposed 'New hook' in Oddewatt, medium to coarse sand $0.1 \mathrm{~m}$ below high tide level, 40-50 cm below the surface (March 2016, 1 individual).

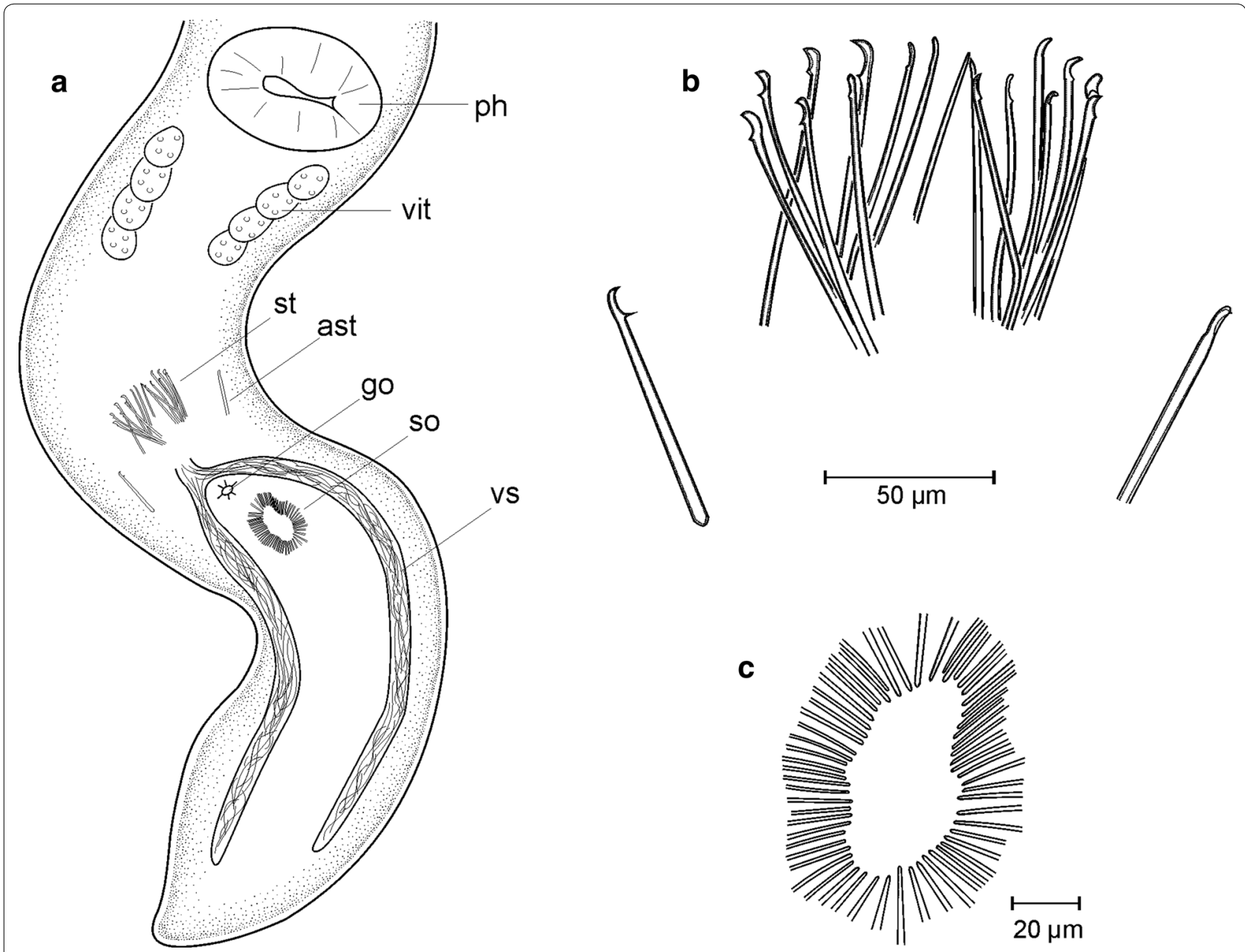

Fig. 2 Coelogynopora multispina n. sp. a Caudal end from life observation. b Needles of the copulatory apparatus. c Solar organ 


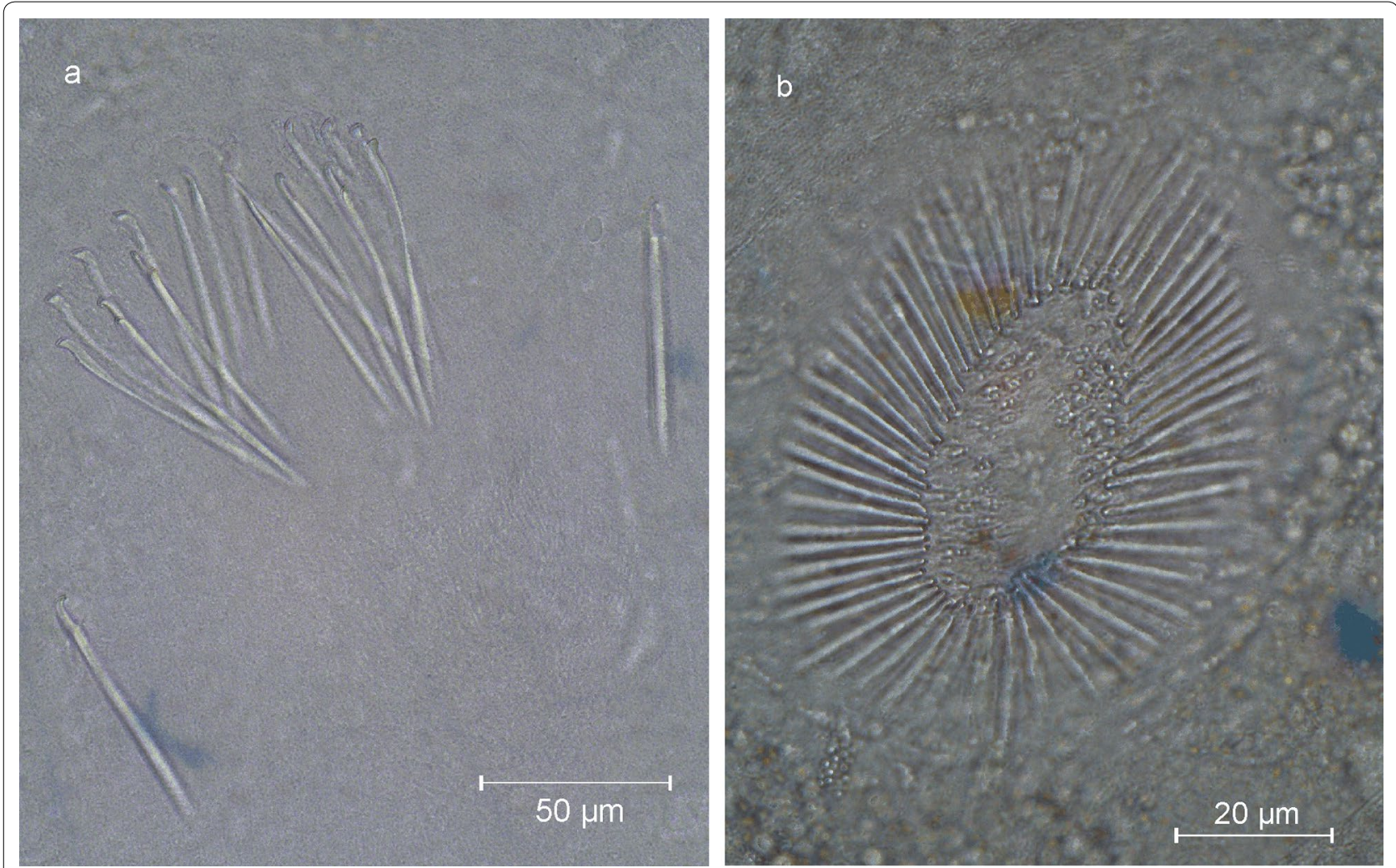

Fig. 3 Coelogynopora multispina n. sp. a Needles of the copulatory apparatus. b Solar organ

Material Live observation, drawings, photographs. The 23 photographs taken during the live observation of the first specimen designated holotype and deposited in the PANGAEA data base: doi:10.1594/PANGAEA.874111.

Etymology The species name refers to the high number of needles in the copulatory and solar organs.

Diagnosis Species of Coelogynopora with a solar organ of some 64 medium-sized needles and a copulatory organ with 16 spines and 2 accessory spines of $70-80 \mu \mathrm{m}$.

Description Life animals are 6-8 $\mathrm{mm}$ long, slightly flattened, and very slender. With roundish epidermal glands up to $20 \mu \mathrm{m}$ in diameter which are most prominent laterally in the first quarter of the body. Pharynx in the beginning of the last quarter of the body, orientated vertically to the body axis. The testis follicles form a median row in the fore body, starting well behind the brain and caudally extending to the germaries. Paired germaries about one body diameter in front of the pharynx, vitellaries in lateral rows, caudally almost reaching the copulatory organ. Very long paired seminal vesicles stretching to the caudal end (i.e. approximatively $1 / 8$ of the body length). Close to the copulatory organ they unite to a short common seminal duct covered by granular vesicles some $50 \mu \mathrm{m}$ before the sclerotic apparatus. Sclerotic part of the copulatory organ with 16 needles surrounding the ejaculatory duct and a pair of caudal accessory needles. The central needles are 70-80 $\mu \mathrm{m}$ long with a curved pointed tip and a triangular projection about $10 \mu \mathrm{m}$ from the tip. The lateral spines are similar in shape and size $(70-75 \mu \mathrm{m})$ but more solid. The female genital duct bears an ovoid solar organ ("Sonnenorgan" in Karling [40]; "ein durch Stachelkranz ausgezeichnetes Reizorgan" in Meixner [47], legend to Fig. 59). In C. multispina the solar organ consists of 64 needles (average needle size $20 \mu \mathrm{m}$, range $18-23 \mu \mathrm{m})$.

Discussion More than 30 species of Coelogynopora have been described so far but only 3 of them have a solar organ. Coelogynopora multispina differs from all of them by the number and sizes of needles and spines in the solar and copulatory organs (Table 5).

\section{Pseudostomum gracilis Westblad 1955 (Fig. 4)}

Locality Sylt Island, List, beach next to the ferry landing, fine- to medium sand in the lower intertidal. May to September, most abundant in August (total 36 individuals). 
Table 5 Number and size of needles in the sclerotic apparatus of the Coelogynopora species with a solar organ

\begin{tabular}{|c|c|c|c|c|}
\hline & C. schulzii & C. solifer & C. visurgis & C. multispina \\
\hline \multicolumn{5}{|l|}{ Solar organ } \\
\hline $\begin{array}{l}\text { Number of } \\
\text { needles }\end{array}$ & 40 & $28-30$ & $25-30$ & 64 \\
\hline $\begin{array}{l}\text { Needle size } \\
(\mu \mathrm{m})\end{array}$ & 40 & 45 & 10 & 20 \\
\hline \multicolumn{5}{|l|}{$\begin{array}{l}\text { Copulatory } \\
\text { organ }\end{array}$} \\
\hline $\begin{array}{l}\text { Number of } \\
\text { spines }\end{array}$ & 9 & 6 & $6-8$ & 16 \\
\hline $\begin{array}{l}\text { Spine size } \\
(\mu \mathrm{m})\end{array}$ & 140 & 110 & $5-7$ & $70-80$ \\
\hline \multicolumn{5}{|l|}{$\begin{array}{l}\text { Accessory } \\
\text { spines }\end{array}$} \\
\hline $\begin{array}{l}\text { Number of } \\
\text { spines }\end{array}$ & 2 & 2 & - & 2 \\
\hline $\begin{array}{l}\text { Spine size } \\
(\mu \mathrm{m})\end{array}$ & 130 & 80 & & $70-75$ \\
\hline Source & Luther [43] & Sopott [73] & $\begin{array}{l}\text { Sopott-Ehlers } \\
\text { [75] }\end{array}$ & \\
\hline
\end{tabular}

Material Live observation, drawings, photographs.

Description Free-swimming animals up to $1.2 \mathrm{~mm}$ long, slender, yellowish opaque. About 1 body diameter from the front end the body is slightly constricted with the mouth and genital opening ventrally and 4 eye spots dorsally. Pharynx weakly muscular. The testis follicles form a uniform mass in mid body. The paired vitellaries are distinctly separated from the single germary, vitelloducts could not be observed. Caudal end with a seminal bursa.

The copulatory organ lies in the caudal third of the body in free-swimming specimens but shortly behind the pharynx in contracted ones. The genital atrium is clearly visible as a relatively broad duct with a small enlargement close to the copulatory organ, presumably the 'small gland' described by Westblad [82]. The copulatory organ consists of a small penis papilla with glands containing very fine secretions and a granular vesicle. The granular vesicle contains coarse secretions proximally and finer secretions distally, often separated by a slight constriction. The seminal vesicle is as large as the granular vesicle. A broad deferent duct stretches from the caudal end of the seminal vesicle alongside the copulatory organ but could not be further tracked towards the testes follicles.

Discussion So far, P. gracilis was only known from 2 preserved specimens found in Strömmarna near Gullmar Fjord, Sweden, in mud from 5 to $10 \mathrm{~m}$ depth [82]. With its single germary that is clearly separated from the vitellaries the species is easily identified, but as pointed out by Westblad [82], the same characters cast some doubt on its classification in the genus Pseudostomum.

Nesite n. gen. (Neodalyellida Willems et al. 2006)

Diagnosis Neodalyellioid with paired germovitellaries and a single testis, genital pore in the caudal body half; stylet complicated with lateral spine-shaped appendages. Type and single species: Nesite ptychoperoides $\mathrm{n}$. sp.

Etymology Acronym for neodalyellioid with single testis.

Nesite ptychoperoides n. sp. (Figs. 5, 6)

Locality Island of Sylt, 'New hook' in Oddewatt north of List (locus typicus, $55.0241^{\circ} \mathrm{N}, 8.4379^{\circ} \mathrm{E}$ ). Medium to coarse shore sand, lower to mid intertidal, surface to $20 \mathrm{~cm}$ sediment depth; 4 individuals found in June 2015, April and May 2016.

Material Life observations including drawings and photographs. The 24 photographs taken during the live observation of one specimen designated holotype and deposited in the PANGAEA data base: doi:10.1594/ PANGAEA.874117.

Etymology The stylet shape reminds to those in the trigonostomid genus Ptychopera.

\section{Diagnosis Currently as Nesite n. gen.}

Description Unpigmented slender organisms, free swimming $0.7-0.8 \mathrm{~mm}$ long and $0.15 \mathrm{~mm}$ wide with broadly rounded body ends. With paired eye pigmentations that are relatively large $(10 \times 15 \mu \mathrm{m})$ and close $(20-30 \mu \mathrm{m})$ to the frontal end. The pharynx doliiformis is $150 \mu \mathrm{m}$ long and $70 \mu \mathrm{m}$ wide and starts $50-70 \mu \mathrm{m}$ from the frontal end; mouth opening subterminally.

The paired germovitellaries are situated laterally at both sides of the body, the vitellarian part already starting besides the pharynx; the most developed egg cells directly join the vitellaria. The germaries, testis, and genital opening are all in the second half of the body. At the beginning of the last quarter of the body, the genital opening leads into a rather wide $(50 \mu \mathrm{m}$ in diameter) genital atrium; glands were seen in the frontal end of the atrium and surrounding the genital pore. No further differentiations of the genital atrium were observed.

In all specimens observed the single testis (about $100 \times 150 \mu \mathrm{m})$ covered the right side germovitellary directly behind mid-body. Only a single deferent duct 

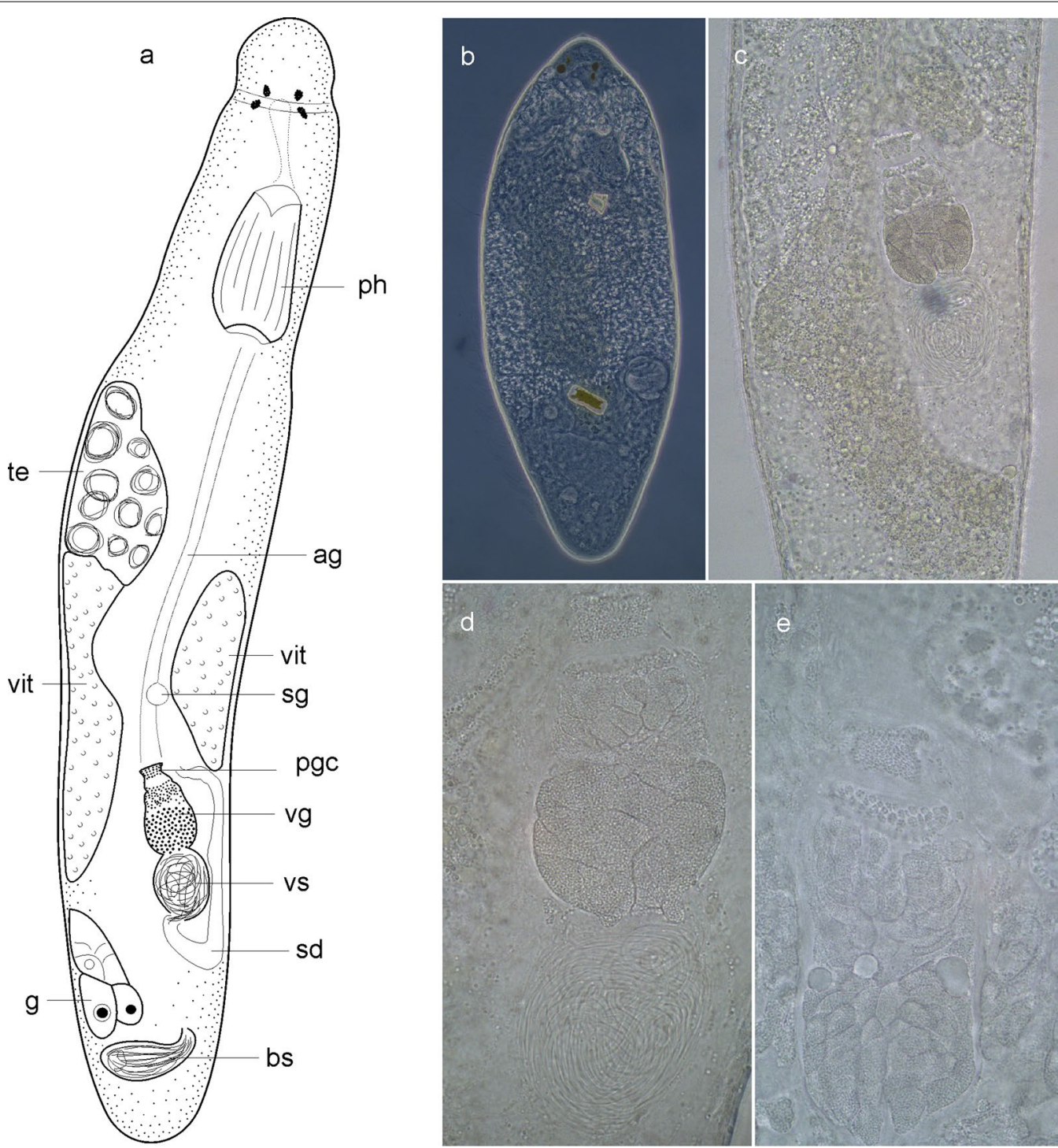

Fig. 4 Pseudostomum gracilis. a Organization from life observation. b Total view. c Hind end with copulatory organ. d, e Copulatory organ with muscular penis, granular vesicles, and seminal vesicle

was observed. The muscular copulatory organ is spherical to slightly ovoid (diameter $50 \mu \mathrm{m}$ to $40 \times 60 \mu \mathrm{m}$ ). Both prostatic glands and the seminal vesicle enter the copulatory bulb proximally. The single seminal vesicle is inversely pear shaped, proximal diameter 20 and $5 \mu \mathrm{m}$ distally, and contains sperm in a well ordered parallel arrangement. The copulatory stylet is rather complicated in shape; basically it is a short wide tube with a one-sided distal hood and two pairs of lateral spine-shaped appendages. The total length of the stylet varied between 67.5 and $71.5 \mu \mathrm{m}$ and the maximum width between 26.5 and $29 \mu \mathrm{m}$ (means of three measurements 69.4 and $27.7 \mu \mathrm{m}$, respectively).
Discussion So far, the combination of paired germovitellaries and a single testis is not known in freeliving species of Neodalyellida. Generally the testes are paired, though they may be partially fused in Baicalellia (but still with paired deferent ducts and paired seminal vesicles) and Haplovejdovskya (but combined with an unpaired germovitellary). Stylets with spineshaped appendages also occur in the genera Kirgisiella, Selimia and Drepanilla but they all have an unpaired germary.

Haplovejdovskya scilliensis Faubel \& Warwick 2005 (Figs. 7, 8) 

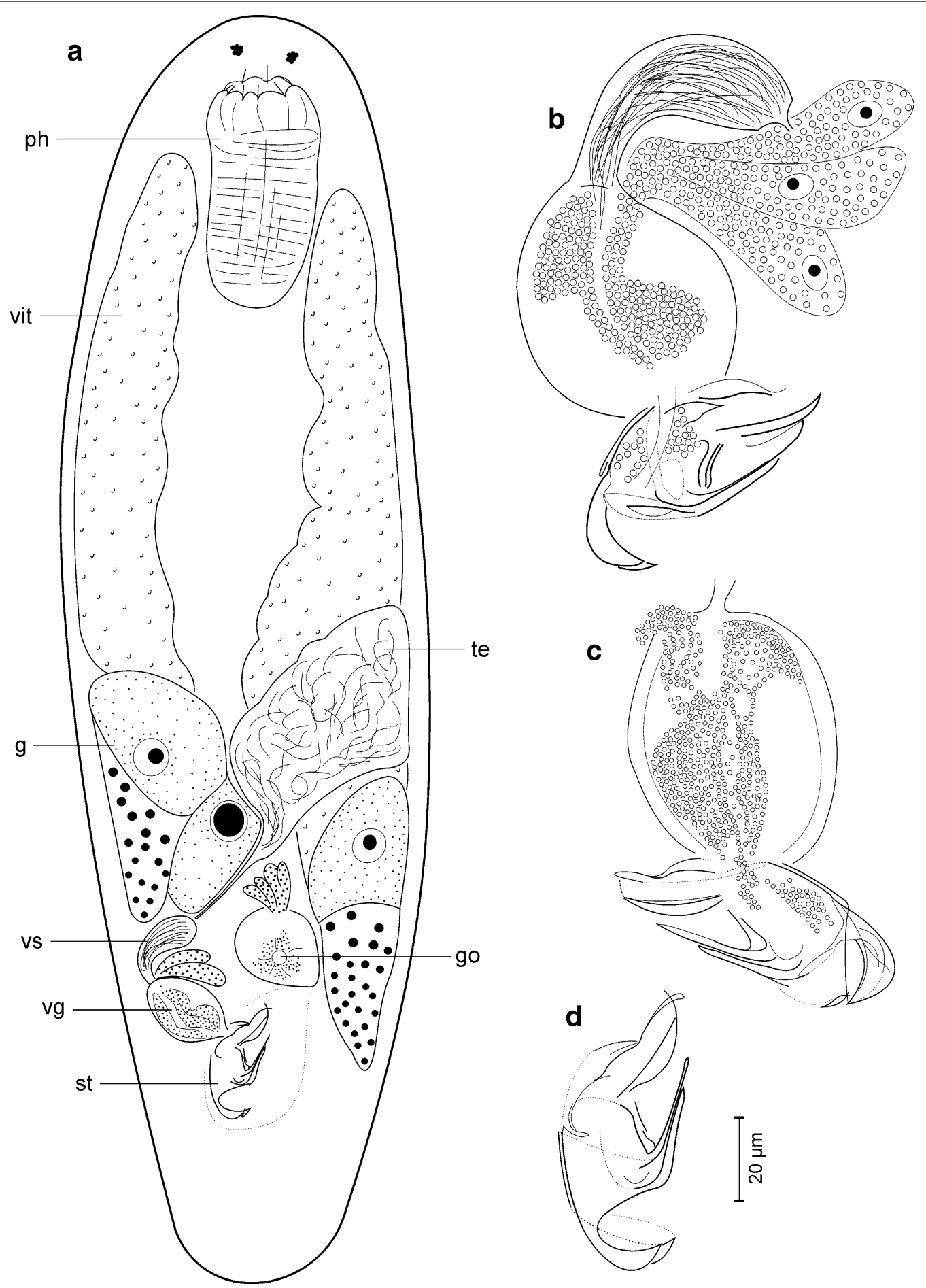

Fig. 5 Nesite ptychoperoides. a Organization from life observation. b, c Copulatory organs from two different specimens. d Stylet

Locality Sylt, Oddewatt near List, lower intertidal sand (June 2015, 8 individuals; August 2015, 2 individuals; June 1988, 2 individuals).
Description Body length of mature organisms 0.6$0.7 \mathrm{~mm}$, front end gently rounded, rear end more triangular, no body pigmentation, no eyes. Pharynx moderately 

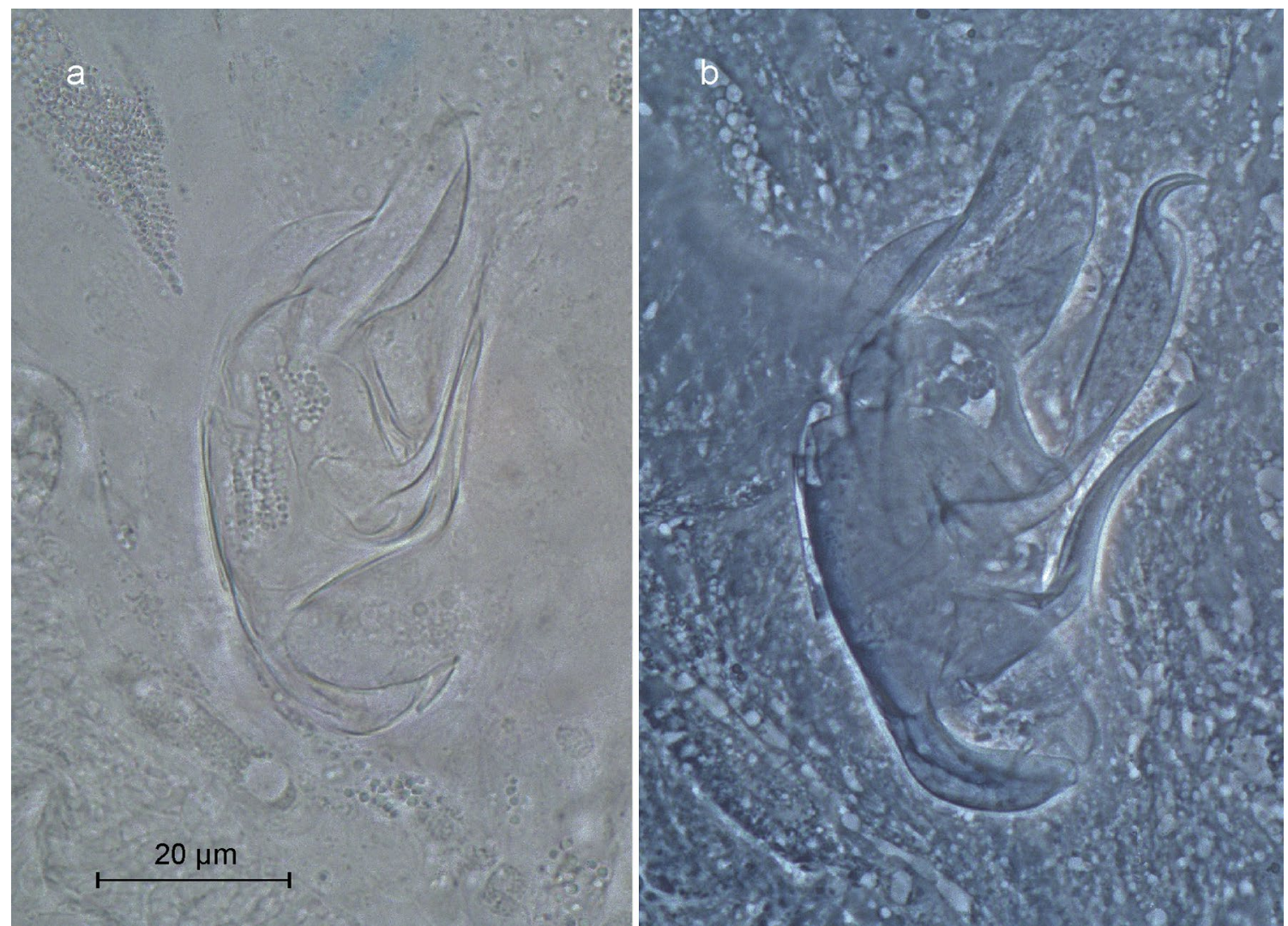

Fig. 6 Nesite ptychoperoides, stylets. a Slightly, b stronger squeezed

large $(85 \times 100 \mu \mathrm{m}$ in a smaller and $100 \times 130 \mu \mathrm{m}$ in a larger specimen), in the front $1 / 5$ of the body, mouth opening subterminally. Besides the pharynx there are paired glands with granular secretions which seem to empty in the pharynx-intestine transition zone.

Male organs Testis unpaired, in the caudal $1 / 3$ of the body, with a single short deferent duct. The muscular copulatory organ is longish with weakly developed musculature and lies next to the testes. The deferent duct enters the copulatory organ in the proximal end. Prostatic glands lie close to the proximal end of the muscular copulatory organ but seem to enter the copulatory organ further distally, at about $1 / 3$ of its length. The proximal $1 / 3$ of the copulatory organ was only filled with sperm, further distally sperm and prostatic secretions were well arranged in parallel rows. The stylet is a short (27.5$28.0 \mu \mathrm{m}$ ) slightly-curved thorn which is broad (9.5$10 \mu \mathrm{m})$ in the proximal third, slender $(3.5 \mu \mathrm{m}$ in diameter) in the middle section, and pointed in the distal $5 \mu \mathrm{m}$.
Sperm and prostatic secretions enter the stylet through a round opening $(8 \mu \mathrm{m})$ in the proximally swollen part of the tube. The entire stylet lies in a thin walled genital bag passing into a genital canal towards the common genital opening.

Female organs The germovitellary consists of a single vitellary positioned in the distal half of the body and a single germary further to the rear end. A short and wide weakly muscular duct connects the germary to the common genital pore. A pair of glands with granular secretions was seen next to the genital pore at the female side, possibly shell glands. A seminal receptacle is positioned in the distal tip of the body.

Discussion The Sylt specimens largely resemble Haplovejdovskya scilliensis Faubel \& Warwick, 2005 but are smaller in body length (Sylt: $0.6-0.7 \mathrm{~mm}$, Scilly Islands: $1.2 \mathrm{~mm}$ ) and stylet length (Sylt: $28 \mu \mathrm{m}$, Scilly Islands: $38 \mu \mathrm{m})$. In the type species of the genus: H. subterranea 

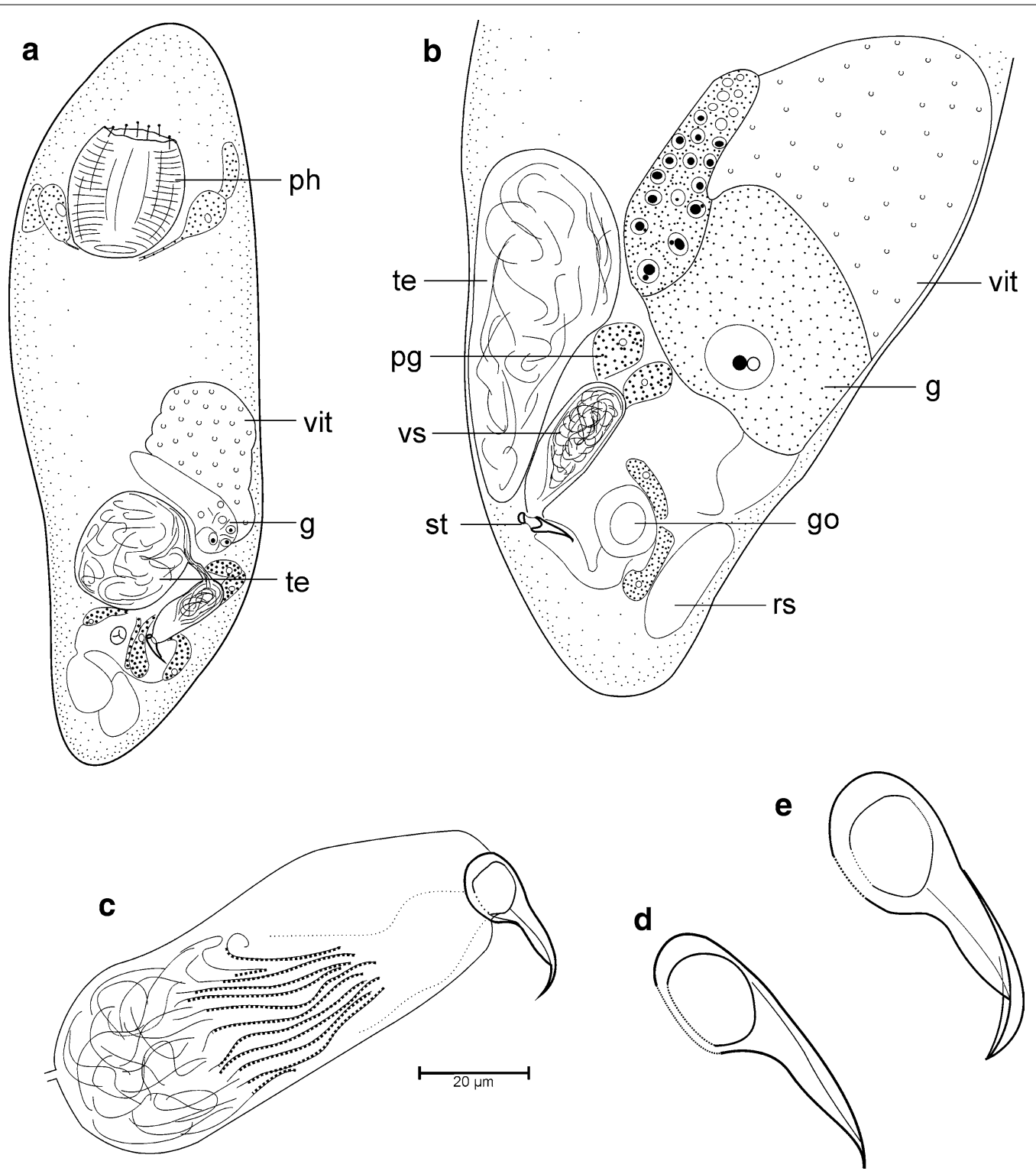

Fig. 7 Haplovejdovskya scilliensis. a, b Organization from life observation. c Copulatory organ. d, e Stylets

Ax, 1954, the testes are fused but the deferent ducts are still paired. This clearly differs in the Sylt specimens with a single deferent duct, but the original description of $H$. scilliensis lacks this information.

Haloplanella magnarhabdita n. sp. (Figs. 9, 10)

Locality Island of Sylt, List, semi-exposed sandy beach next to the ferry landing ('Hausstrand', type locality: $55.0154^{\circ} \mathrm{N}, 8.4361^{\circ} \mathrm{E}$ ), entire beach slope from the bent to mean high tide level but always in larger sediment depth (10-40 $\mathrm{cm}$ below the surface in the lower beach and $40-60 \mathrm{~cm}$ near high tide level. Mature individuals were only found in November and December 2015 (12 individuals).
Material Live observation, drawings, photographs. The 11 photographs taken during the live observation of one specimen are designated holotype and deposited in the PANGAEA data base: doi:10.1594/ PANGAEA.874114.

Etymology The species name refers to the extraordinarily-large frontal rhabdites.

Diagnosis Species of Haloplanella with extraordinarily large frontal rhabdites, a stylet with a wide proximal and a narrow distal part, the latter surrounded by a shoe shaped external lamina. 


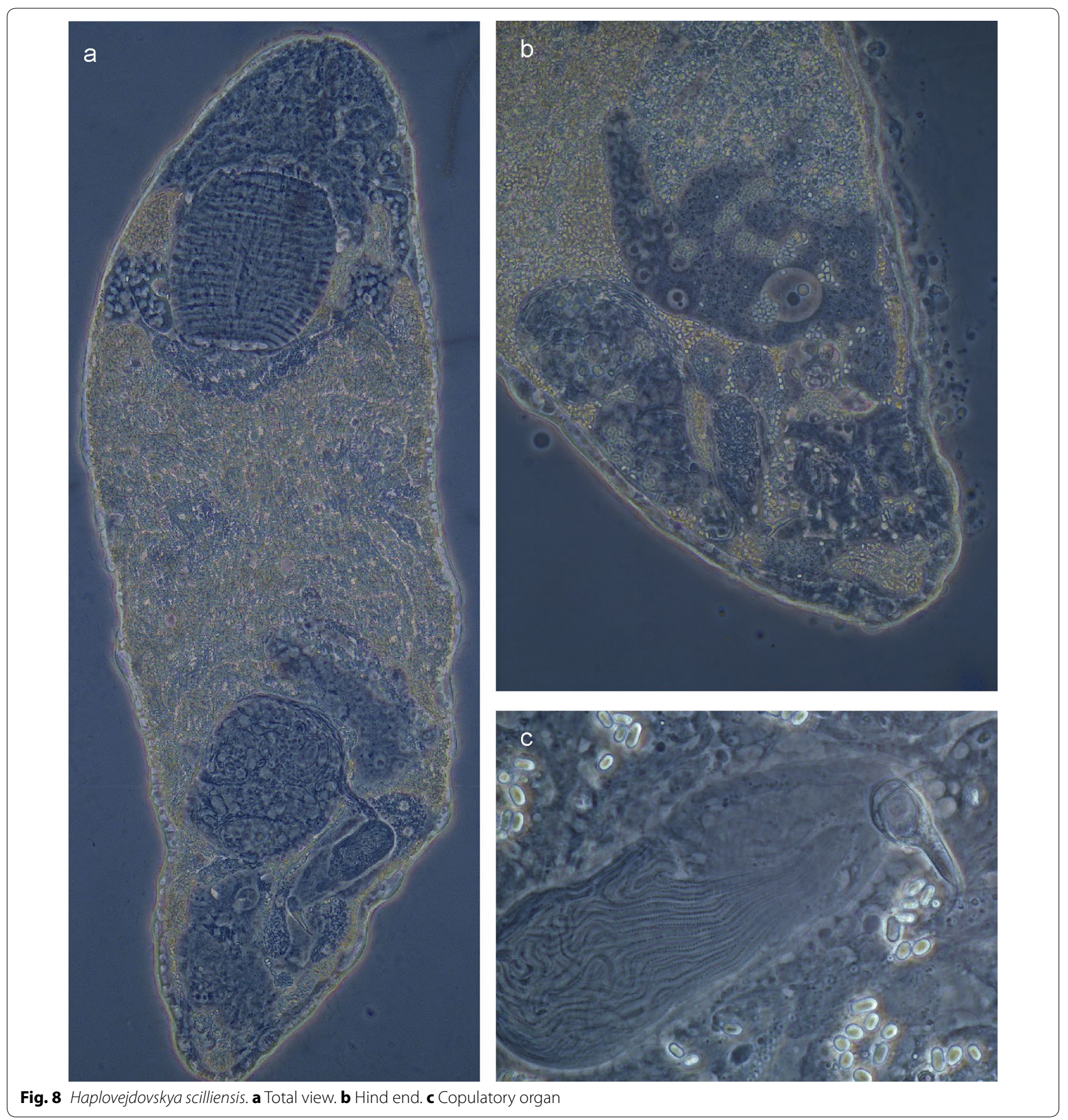

Description Medium-sized slender organisms (free swimming $0.6-1.2 \mathrm{~mm}=$ smallest specimen with a stylet to largest observed), without eye pigmentations. Pharynx in the beginning of the second half of the body. Caudal end gently rounded, frontal end broadly rounded and with large rhabdites in a median line. The paired rhabdites-building frontal glands may stretch back to the pharynx. Length of the rhabdites is $20-25 \mu \mathrm{m}$ in the caudal part of the frontal glands and up to $40 \mu \mathrm{m}$ near the frontal end. 


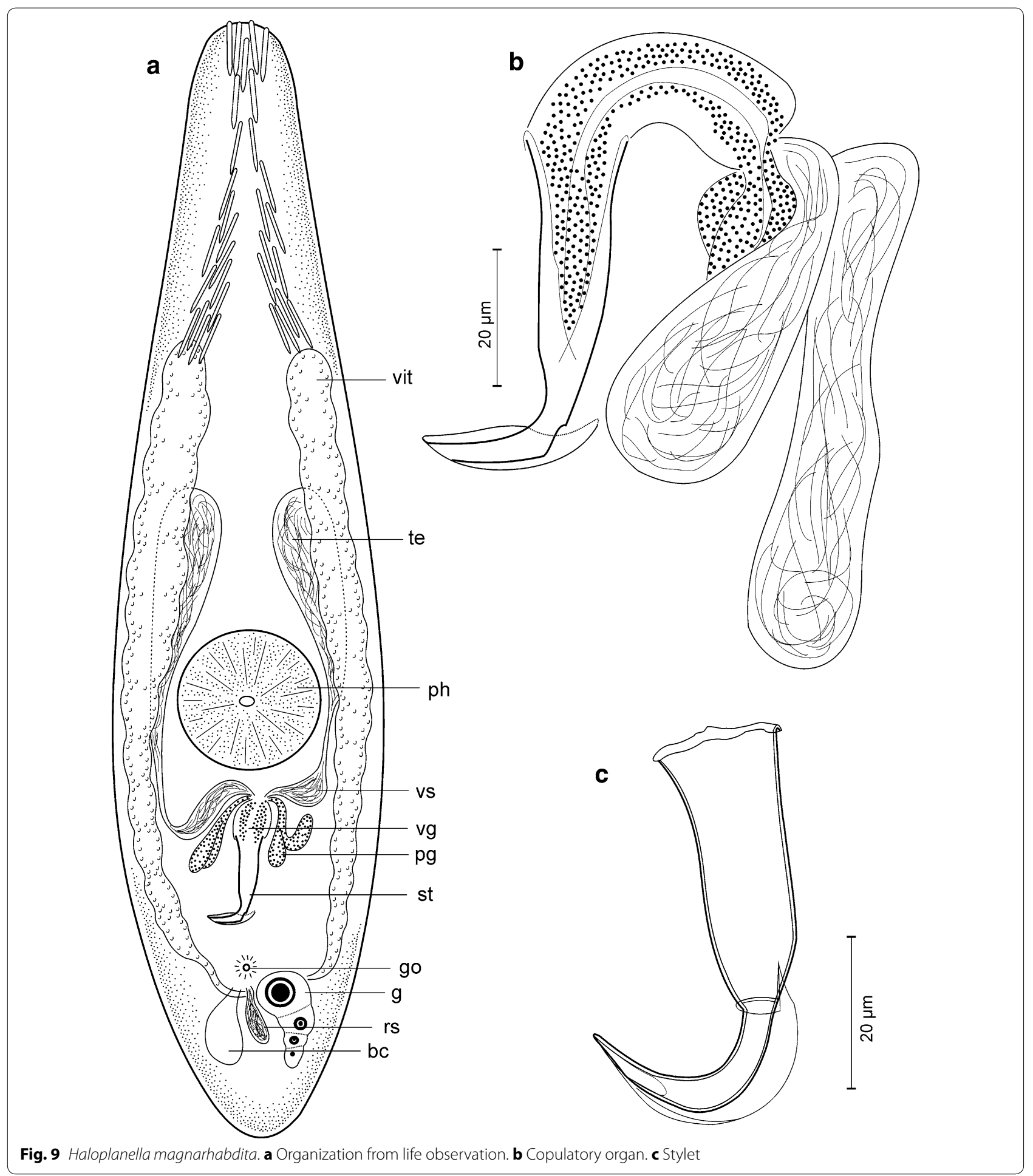

Paired testes situated laterally before the pharynx, the deferent ducts swollen to seminal vesicles before separately entering the copulatory organ together with prostatic glands. Paired vitellaries laterally in the body, from the second quarter of body length almost to the end of the body. Single germary in the body end, accompanied by two longish cavities, one filled with sperm (seminal receptacle) and the other apparently empty (copulatory 

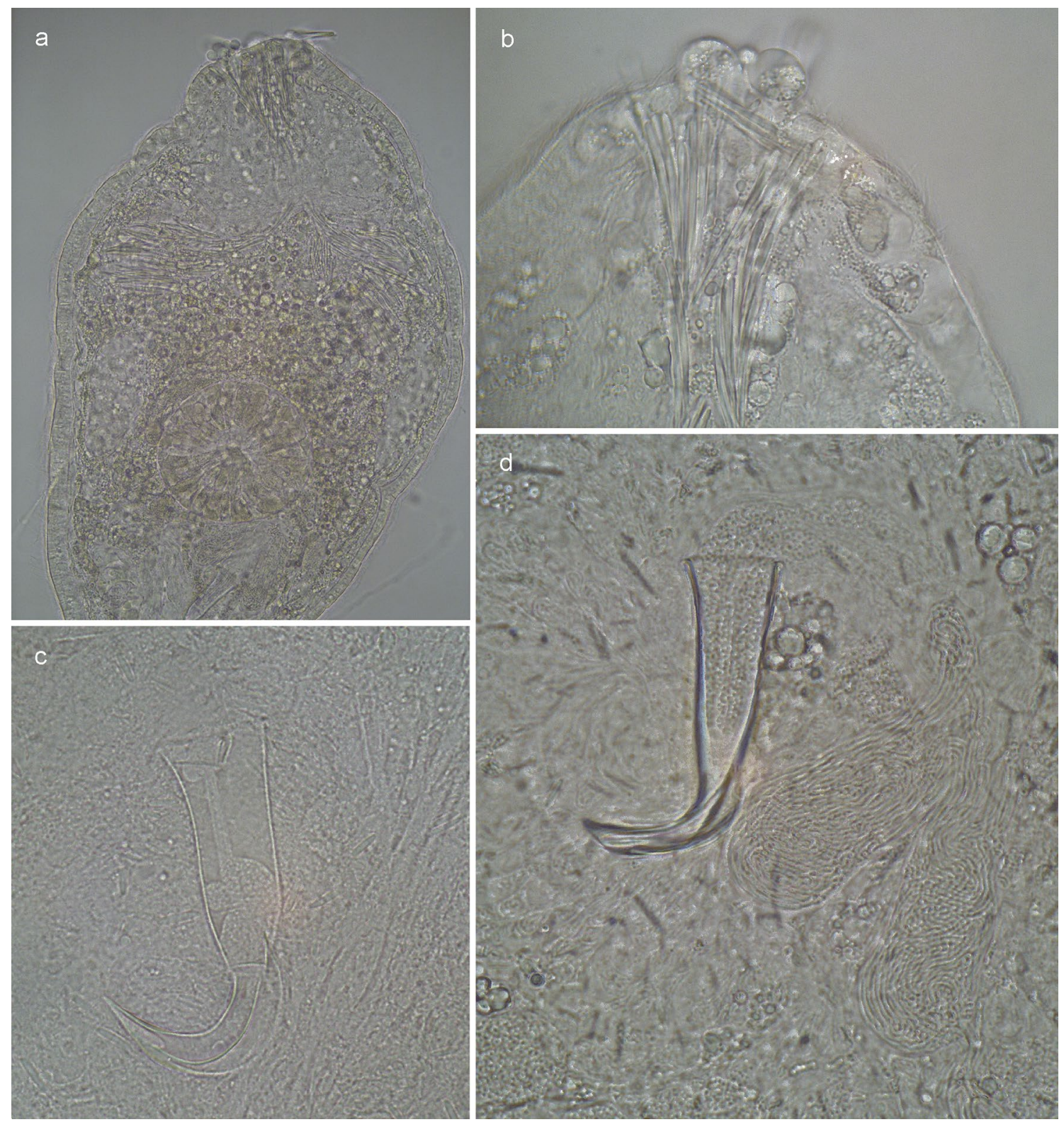

Fig. 10 Haloplanella magnarhabdita. a Total. b Front end with rhabdites. c Stylet. d Copulatory organ

bursa). Genital opening between the germary and the stylet.

The stylet (total length $50-52 \mu \mathrm{m}$ in 4 individuals) is a tube of $11 \mu \mathrm{m}$ diameter in the middle and a slightly enlarged $(12.5-13.0 \mu \mathrm{m})$ proximal opening. In the beginning of the distal half the tube narrows strongly to $4 \mu \mathrm{m}$ and then bends to a semicircular curve with the oval stylet opening at the tip. The narrow part of the tube is partly surrounded by a curved lamina shaped like a shoe.
Discussion Haloplanella magnarhabdita fits the genus diagnosis given by Ehlers and Sopott-Ehlers [23]. Specific differences from the other species described so far are (1) the stylet consisting of a wide proximal and a narrow distal part with a transition in diameter in a very narrow zone-in all other species the stylet tube narrows continuously over its entire length; (2) the shoe-shaped lamina surrounding the distal part of the stylet. A similar-sized external lamina only occurs in $H$. carolinensis 
Ax 2008 while the lamina in H. obtusituba Luther 1946 is very small; (3) the extraordinary size of the frontal rhabdites.

Haloplanella spiralis n. sp. (Fig. 11)

Locality Island of Sylt, List, semi-exposed sandy beach next to the ferry landing (type locality: $55.0155^{\circ} \mathrm{N}$, $8.4364^{\circ} \mathrm{E}$ ). (1) Mean high tide level, in $50-70 \mathrm{~cm}$ sediment depth (September 2015, 3 individuals). (2) $0.5 \mathrm{~m}$ below mean high tide level, in $30-40 \mathrm{~cm}$ sediment depth (September 2015, 1 individual).

Material Live observation, drawings, photographs. The 10 photographs taken during the live observation of one specimen designated holotype and deposited in the PANGAEA data base: doi:10.1594/PANGAEA.874115.

Etymology The species name refers to the corkscrew shape of the stylet.

Diagnosis Species of Haloplanella with a corkscrewshaped stylet with a weak external lamina in the distal end.

Description Small-sized slender organisms (free swimming 0.5-0.7 mm long) without eye pigmentations. Caudal end gently rounded, frontal end broadly rounded and with faint rhabdites. Pharynx in the beginning of the second half of the body, genital opening ventrally behind the stylet.

Paired testes laterally before the pharynx, the deferent ducts swollen to seminal vesicles before separately entering the copulatory organ together with prostatic glands. Paired vitellaries laterally in the body, from the second quarter of body length almost to the end of the body. Though the vitellaries were already well developed I could only see a bright spot in the in the body end where the germary is expected. Likewise, the seminal receptacle and copulatory bursa could not positively be identified. The stylet (total length $42.7 \mu \mathrm{m}$ ) is a continuously-narrowing tube of $11 \mu \mathrm{m}$ proximal and $2.6 \mu \mathrm{m}$ distal diameter (a single measurement only). It winds like a corkscrew with a small deflection proximally and a very strong one distally to end in a semicircular curve with the stylet opening at the tip. The distal half of the tube is partly surrounded by a weak external lamina.

Discussion Though all structures observed fit the genus diagnosis of Haloplanella given by Ehlers and Sopott-Ehlers [23] the female organs were not sufficiently developed for a positive classification with the genus,

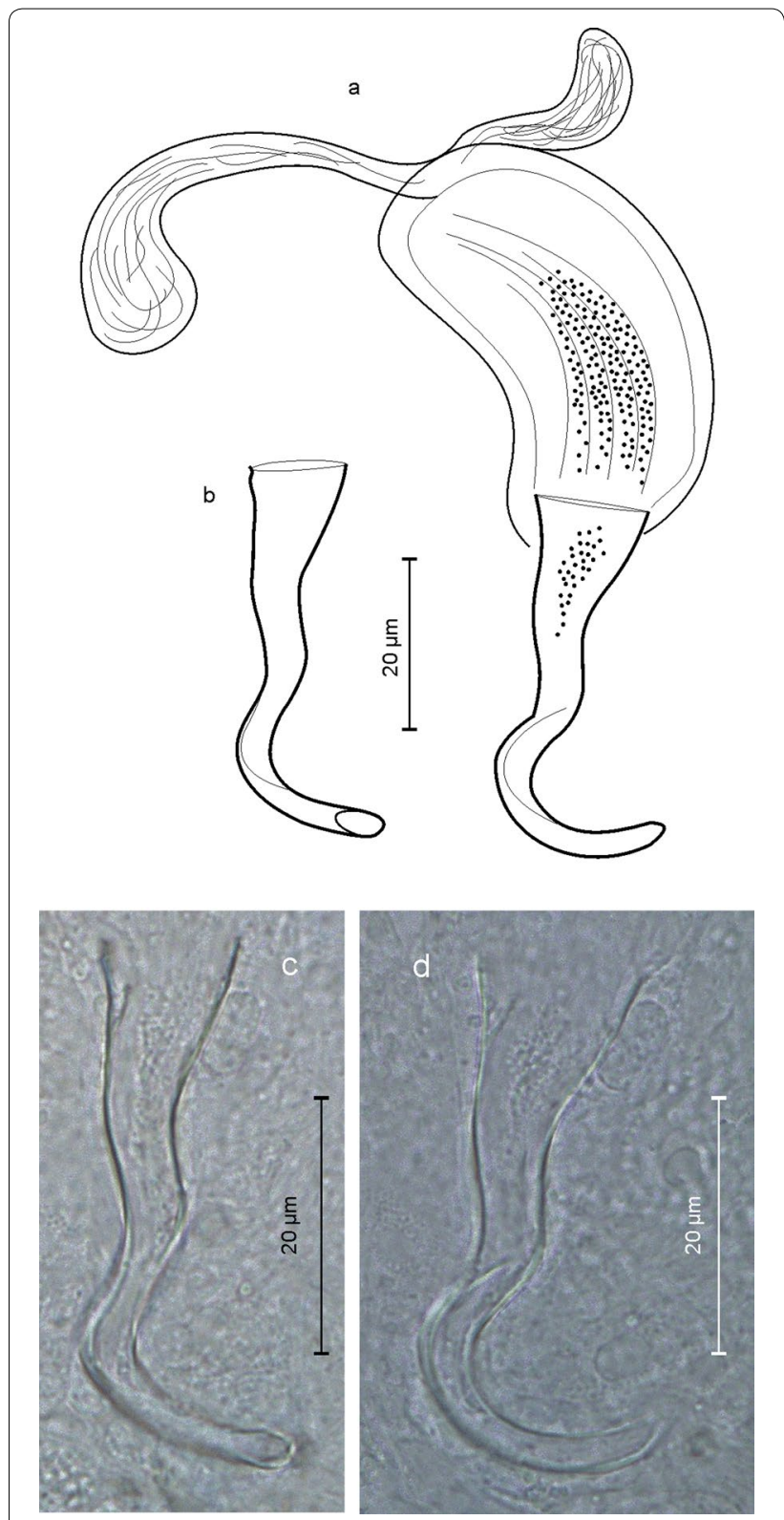

Fig. 11 Haloplanella spiralis. a Copulatory organ. b-d Stylet

so the classification is provisional. H. spiralis is the only species in the genus with a spiral-shaped stylet. A weak distortion of the tube only occurs in $H$. conversa Ehlers \& Sopott-Ehlers, 1989 but not combined with the wide semicircular shape in the distal part.

Moevenbergia oculifagi n. sp. (Fig. 12)

Localities Island of Sylt. Previous findings (Moevenbergia oculofagi n.n.): (1) Königshafen mid-tidal sand 

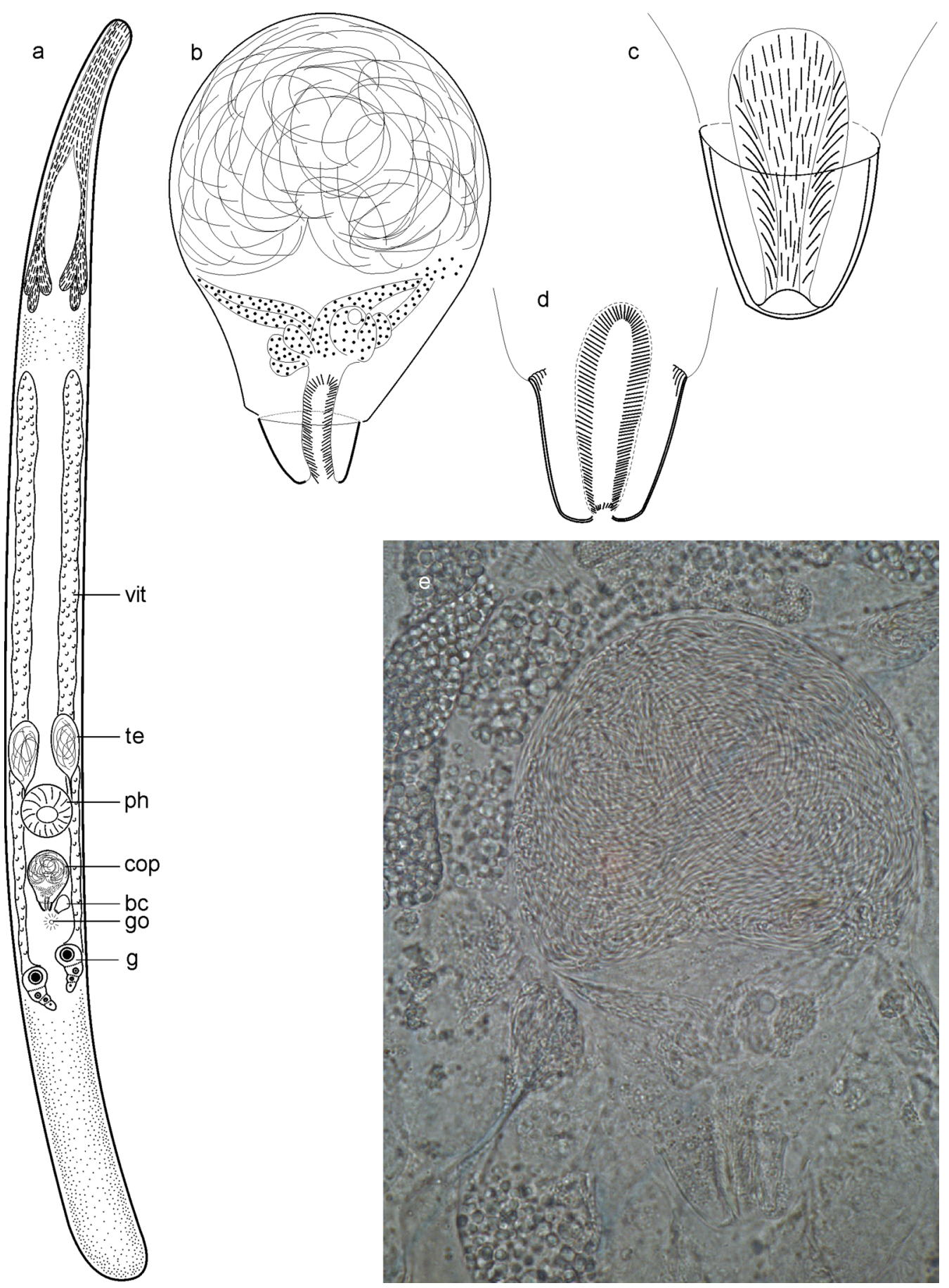

Fig. 12 Moevenbergia oculofagi. a Organization from life observation. b, e Copulatory organ. c, d Stylet

flats with burrows of lugworms Arenicola marina [61, $62,64,66]$. (2) Königshafen, sand flat close to high tide level [36]. (3) Rantum, medium sand close to high tide level [36]. (4) Königshafen, from lower intertidal to shallow subtidal (2-3 m below mean low tide level) mud and muddy sand [2]. New findings: (5) Island of Sylt, subtidal $(0.5 \mathrm{~m})$ fine sand east of Oddewatt (May 2015, 3 individuals). (6) Island of Sylt, List, lower intertidal medium sand $300 \mathrm{~m}$ south of the ferry landing (June 2015, 1 individual). 
Material Live observation, drawings and photographs; including drawings from previous findings by Karsten Reise. The specimen from locality 6 was studied in most detail and the 14 photographs taken during live observation on it are designated holotype and deposited in the PANGAEA data base: doi:10.1594/PANGAEA.874118. Type locality: Island of Sylt, List $\left(55.0152^{\circ} \mathrm{N}, 8.4364^{\circ} \mathrm{E}\right)$, lower intertidal medium sand.

Etymology The species name was adopted from the nomen nudum used in previous publications (see above).

Diagnosis Species of Moevenbergia with strong frontal adenal rhabdites and a copulatory organ with long (30$34 \mu \mathrm{m})$ cirrus.

Description Very slender organisms, free swimming 1.8 to $2.5 \mathrm{~mm}$ long and $10-12$ times as long as wide. Frontal end only half the diameter of the rest of the body and almost completely filled with adenal rhabdites, which are straight rods of 10-12 $\mu \mathrm{m}$ length tapering at both ends. Dermal rhabdites of 3-4 $\mu \mathrm{m}$ length are scattered over the entire body. Pharynx in the end of the second third of the body, diameter 120-140 $\mu \mathrm{m}$. Caudal end nearly semicircular, no pigmented eyes.

Paired testes directly before, copulatory organ directly behind the pharynx. The copulatory organ is reversed pear-shaped, total length about $100 \mu \mathrm{m}$, proximally with a spherical seminal vesicle (diameter $70 \mu \mathrm{m}$ ) and granular prostatic secretions distally. The deferent seminal ducts are only slightly enlarged before entering the copulatory organ dorsally. External prostatic glands were not observed. The distal part of the copulatory organ consists of a slightly tapering tube of $18-22 \mu \mathrm{m}$ length and an inner cirrus of 30-34 $\mu \mathrm{m}$ length. The cirrus spines are very thin and positioned very close to each other.

Paired vitellaries situated laterally in the body, from the beginning of the second to the end of the third quarter of the body. Paired germaries directly fused to the caudal end of the vitellaries leaving the last quarter of the body without reproductive organs. Genital opening shortly behind the copulatory organ, genital atrium relatively small. Copulatory bursa $40-50 \mu \mathrm{m}$ long, apparently with a hardened inner wall, directly besides the copulatory organ. Up to now, seminal receptacles could not be observed in life organisms.

Discussion $M$. oculofagi fits the genus diagnosis of Moevenbergia. However, some characters have not yet been observed, such as the existence of paired seminal receptacles or the pharynx ciliation. Therefore the classification of the new species in Moevenbergia is provisional, mainly following the previous references. Within the genus, M. oculofagi differs from M. una Armonies \& Hellwig 1987 by the dimensions of the hardened parts of the copulatory organ (about twice the size in $\mathrm{M}$. oculofagi) and by the strong development of the frontal organ.

Coronhelmis inaequalis n. sp. (Figs. 13, 14)

Localities (1) Island of Sylt, List, lower intertidal medium sand $200 \mathrm{~m}$ south of the ferry landing (type locality, $55.0152^{\circ} \mathrm{N}, 8.4364^{\circ} \mathrm{E}$; June 2015,1 individual). (2) Island of Sylt, Oddewatt north of List, fine sand at mean low tide level (August 2015, 1 individual). (3) Island of Sylt, Oddewatt north of List, shallow subtidal $(0.5 \mathrm{~m})$ fine sand (May 2016, 6 individuals).

Material Live observation, drawings and photographs. The 18 photographs taken during live observation of the first specimen designated holotype and deposited in the PANGAEA data base: doi:10.1594/PANGAEA.874112.

Etymology The species name refers to the inequality of spines in the copulatory organ.

Diagnosis Species of Coronhelmis with different shaped and sized stylet spines.

Description Medium sized slender organisms, free swimming 0.7 to $1.0 \mathrm{~mm}$ long and with a pointed frontal and gently rounded hind end, without eye pigmentations. Frontal organ moderately developed, stretching back to the first sixth of the body in resting specimen and the first quarter in free swimming ones. Pharynx relatively small (diameter $70 \mu \mathrm{m}$ ), positioned in the end of the first half of the body.

Paired testes fused before the pharynx, but still with paired deferent ducts. Copulatory organ positioned directly behind the pharynx, with proximal seminal vesicles and distal granular prostatic secretions, prostatic glands faint. Paired vitellaries laterally in the body, starting shortly before the pharynx and extending almost to the end of the body. Paired germaries in the very end of the body in smaller organisms and in the last quarter of the body in larger ones. Genital opening shortly behind the copulatory organ, a small copulatory bursa (containing some sperms in most of the specimens) besides the copulatory organ. Seminal receptacle extraordinarily large, between the genital opening and the germaries.

Stylet without a proximal tube, merely a narrow ring $30 \mu \mathrm{m}$ in diameter bearing spines. Two of the spines are large $(15 \mu \mathrm{m}$ in length and $9 \mu \mathrm{m}$ in basal width) and with a curved tip, one spine is medium sized $(10 \mu \mathrm{m}$ length and $6 \mu \mathrm{m}$ basal width). These 3 spines form the dorsal 


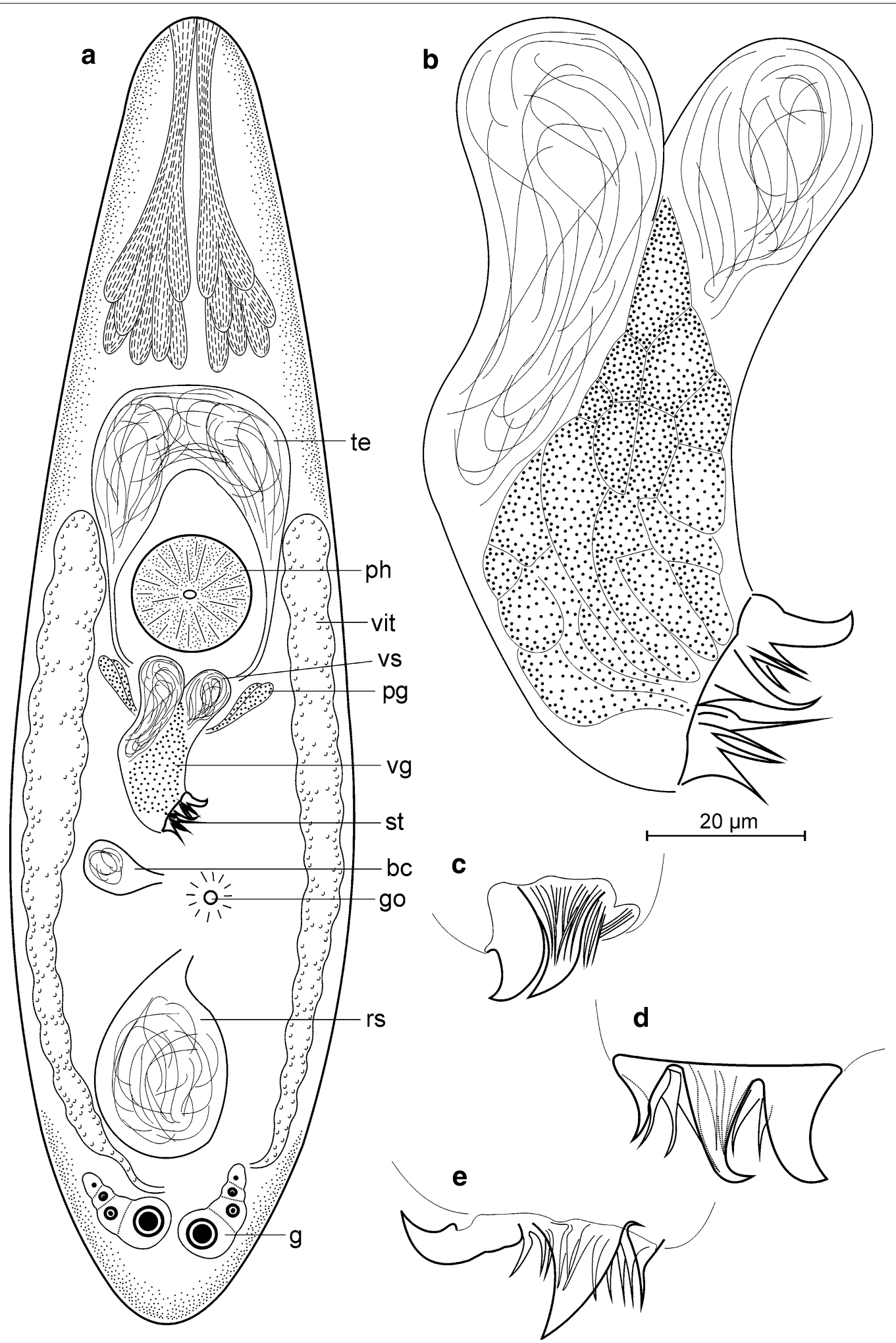

Fig. 13 Coronhelmis inaequalis. a Organization from life observation. b Copulatory organ. c-e Stylet spines, increasing squeezing pressure 


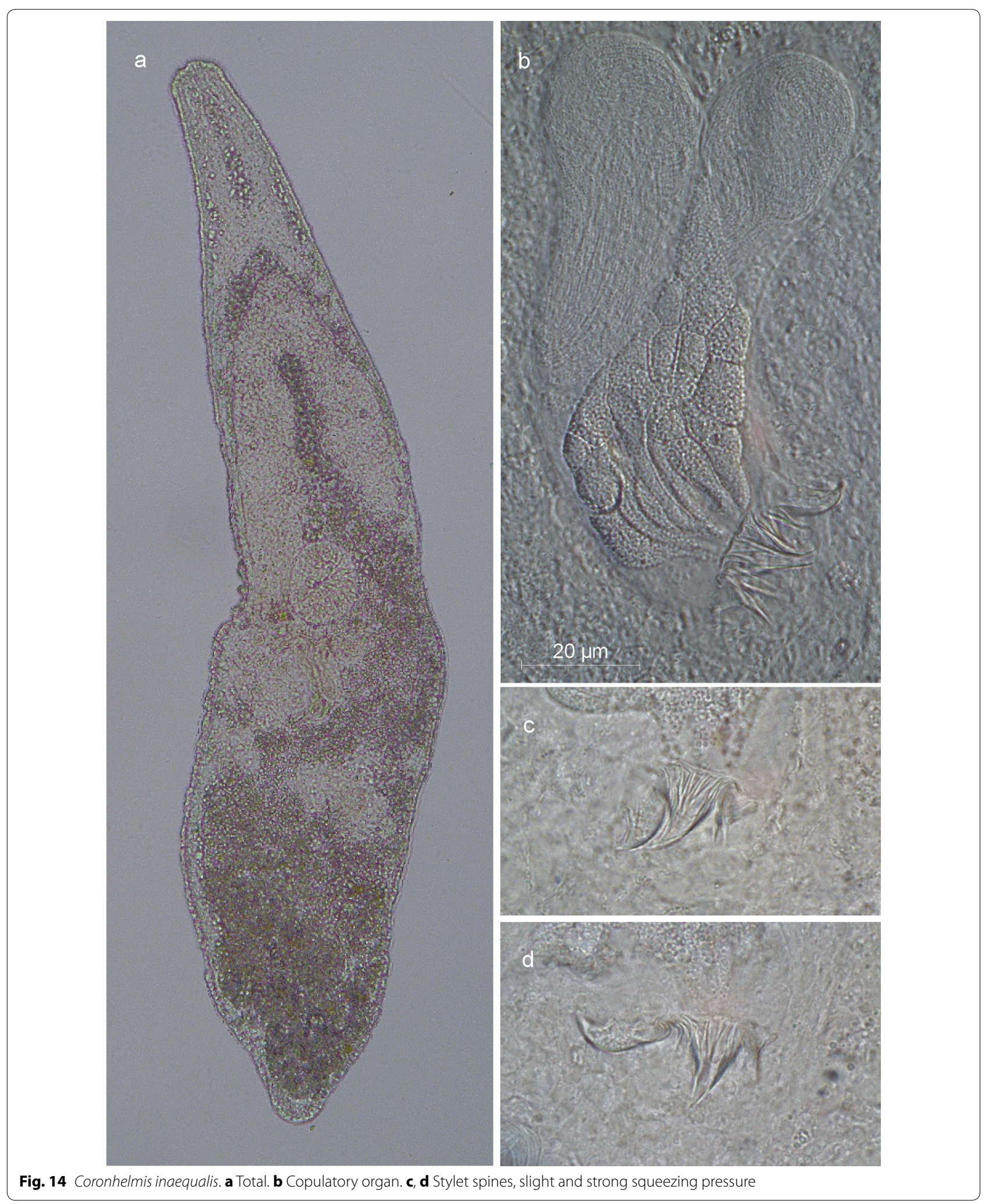


part of the ring. The ventral part consists of about 10 slender spines also about $10 \mu \mathrm{m}$ long but only $2-3 \mu \mathrm{m}$ in basal width. With low coverslip compression, the smaller spines are all hidden below the two large ones. Strong coverslip compression causes one of the large spines to bend sharply outside.

Discussion The lack of a proximal tubiform part of the stylet ("manschette") combines $C$. inaequalis with $C$. exiguus Ax, 1994 and C. inornatus Ehlers, 1974 and separates these 3 species from all other ones of the genus (see Table 1 in [87]. So far, C. inaequalis is the only species of the genus with differently shaped stylet spines. Possibly the proximal fusion of the testes is another autapomorphy although the testes seem to be very close to each other in all of the species described so far.

Proxenetes inflatus den Hartog 1966 (Fig. 15)

Localities Island of Sylt, Oddewatt north of List, lower slope of 'New hook' (May 2015, 1 individual). Previously only known from sublittoral $(40 \mathrm{~m})$ soft mud of Gullmar Fjord, Sweden (September 1951, 1 individual, [33].

Material Live observation, drawings and photographs.

Discussion The specimen was easily recognized by the widely inflated cuticular mantle around the stylet. Distally the mantle border is finely serrated. The bursal appendage bears an additional cuticular ring with two lateral spines that are not mentioned in the original description.

Diascorhynchus falconis n. sp. (Figs. 16, 17)

Locality Island of Sylt, New hook in Oddewatt (type locality: $\left.55.0241^{\circ} \mathrm{N}, 8.4392^{\circ} \mathrm{E}\right), 0.4 \mathrm{~m}$ below mean high tide level in 10-20 cm sediment depth (June 2016, 2 individuals).

Material Live observation, drawings and photographs. The 47 photographs taken during live observation of the first specimen designated holotype and deposited in the PANGAEA data base: doi:10.1594/PANGAEA.874113.
Etymology The stylet shape reminds to the bill of a falcon.

Diagnosis Species of Diascorhynchus with a small stylet with lateral extension at the distal tip, bursal mouthpiece not hardened.

Description Slender organisms, free swimming $1.5 \mathrm{~mm}$ long, unpigmented, without eyes. Anterior end equipped with a few sensory hairs, proboscis armed with 2 hooks of $38 \mu \mathrm{m}$ length but differing in shape. The paired proboscis gland sacs are $250 \mu \mathrm{m}$ long, with a strong muscle layer and distally with voluminous gland cells. The hind end is triangular in shape and filled with gland cells that are possibly equivalent to the "Haftdrüsenkomplex" described by Karling (1949) for D. serpens.

The general organization is as in the other species of the genus: paired vitellaries laterally in the body, single germary in the hind end, side by side with a seminal bursa. Testes in a median row in the fore body, number of follicles (4-6) not distinct, with a single deferent duct that splits into two branches before the pharynx that enlarge into longish seminal vesicles behind the pharynx. Copulatory organ small and nearly spherical, genital opening between the copulatory organ and the germary, genital atrium well developed. The stylet is a funnel of $20 \mu \mathrm{m}$ length with a distorted tip besides the distal opening, its shape reminds to the bill of a falcon. Deferent duct of the seminal bursa without a hardened bursal piece but with a longish sphincter.

Discussion Currently the genus Diascorhynchus comprises 7 valid species, D. borealis Meixner 1928, D. bucina Ehlers \& Ehlers 1980, D. caligulatus Ax 1959, D. glandulosus (Beauchamp 1927), D. lappvikensis Karling 1963, D. rubrus Boaden 1963, and D. serpens Karling 1949. They all differ in the shape and size of the stylets. D. falconis has a very small stylet (comparable only to D. lappvikensis) while the shape of the stylet is closest to D. bucina and $D$. caligulatus (only in these three species the stylet bears a lateral extension at the distal tip). The lack of a hardened bursal mouthpiece in $D$. falconis is unique in the genus. 

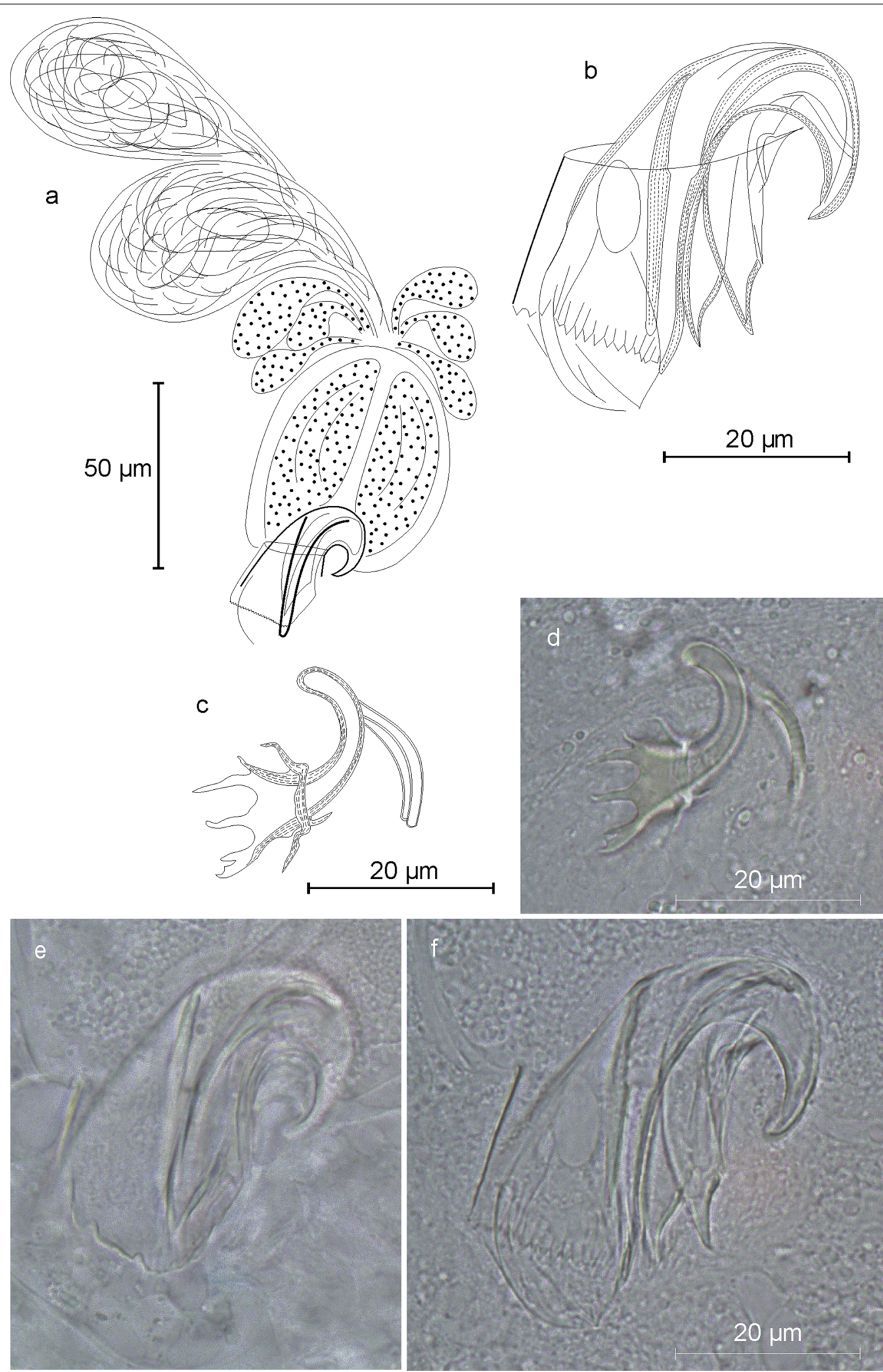

Fig. 15 Proxenetes inflatus. a Copulatory organ. b, e, f Stylet. c, d Bursal appendage 

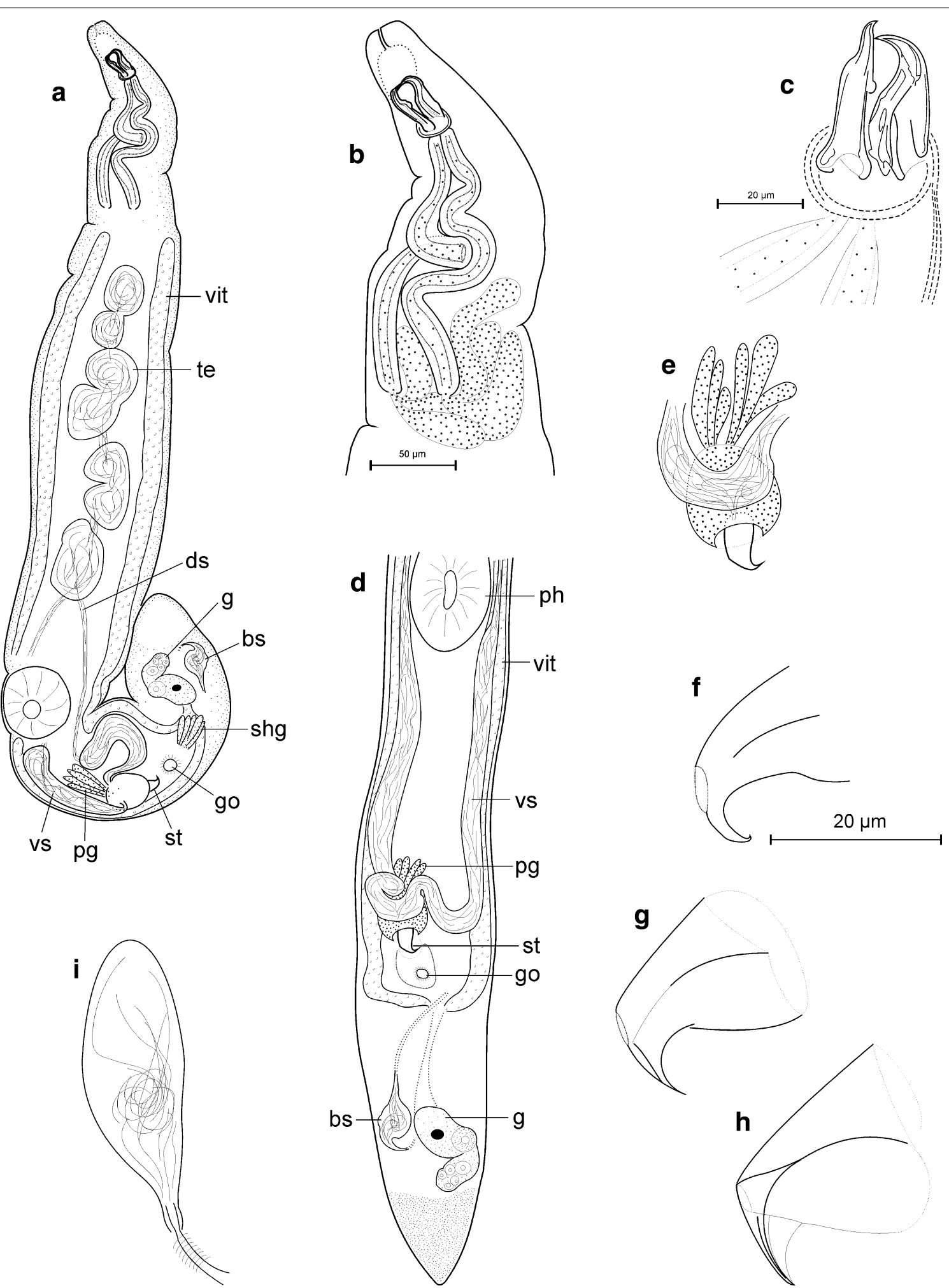

pg
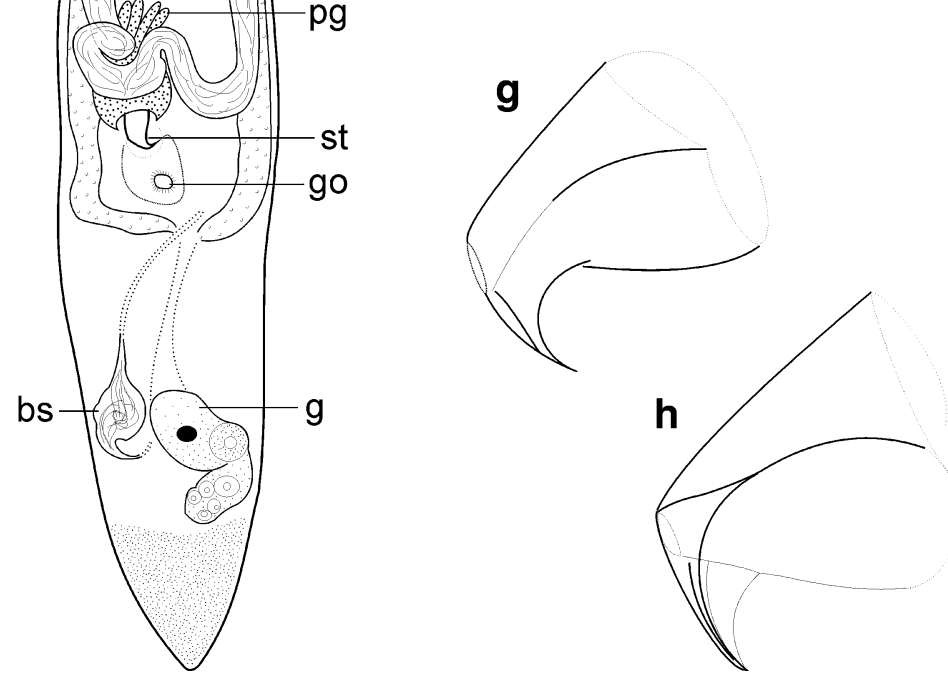

Fig. 16 Diascorhynchus falconis, organization from life observation. a Total view. b Front end with proboscis, muscular proboscis gland sacs, and gland cells. c Proboscis. d Hind end. e Copulatory organ. f-h Stylet. i Seminal bursa with deferent duct 


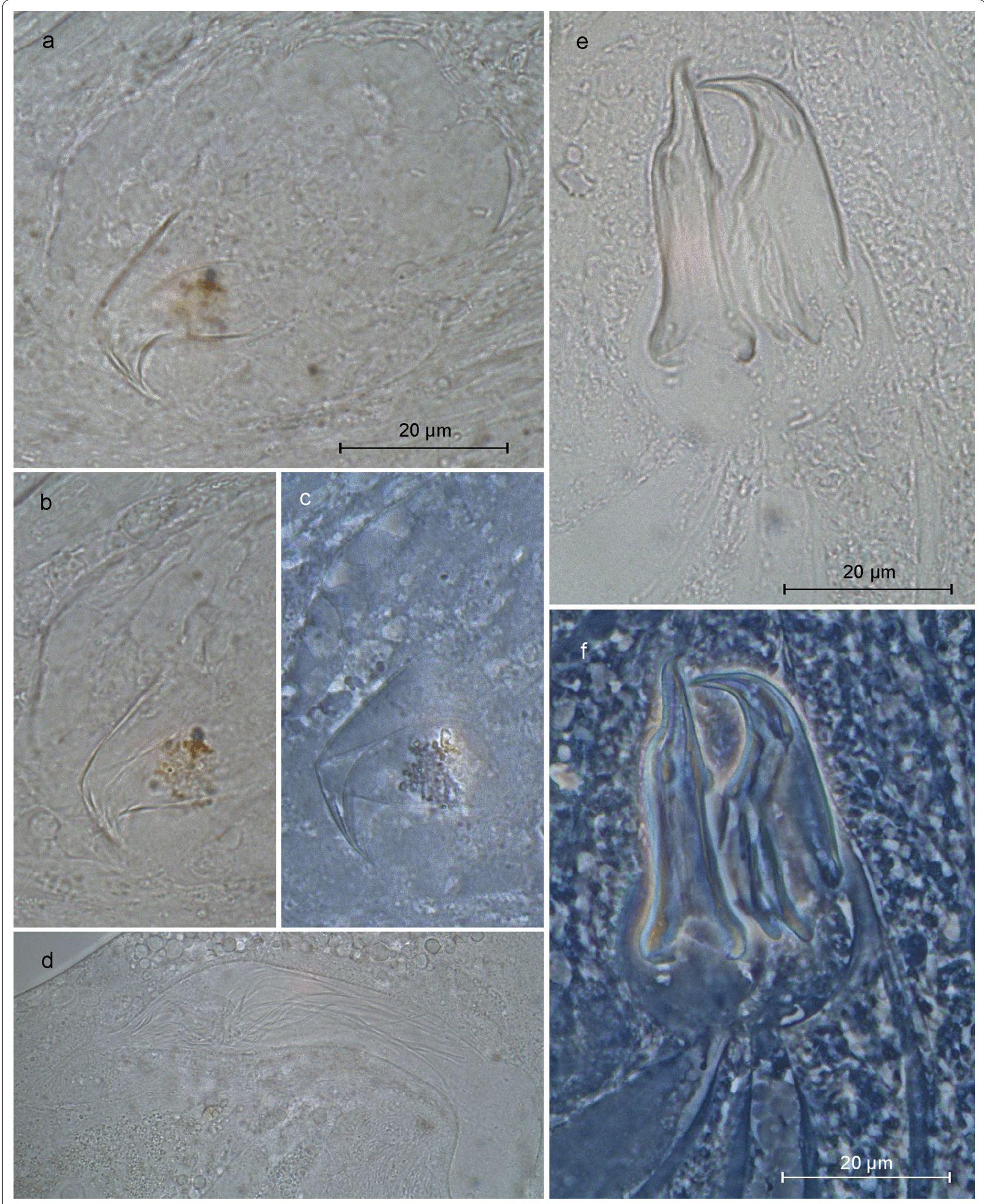

Fig. 17 Diascorhynchus falconis. a-c Stylet. d Seminal bursa with deferent duct. e-f Proboscis 


\section{Abbreviations}

ag: genital atrium; ast: accessory stylet; bc: copulatory bursa; bs: seminal bursa; g: germary; go: genital opening; pg: prostatic glands; pgc: penis papilla with glands; ph: pharynx; rs: seminal receptacle; sd: seminal duct; sg: small gland; shg: shell glands; so: solar organ; st: stylet; te: testis; vg: prostatic vesicle; vit: vitellary; vs: seminal vesicle.

\section{Acknowledgements}

Many thanks to Karsten Reise for providing his personal records on platyhelminth species and improving a former version of the manuscript by critical comments.

\section{Competing interests}

The author declares that he has no competing interests.

\section{Availability of data and materials}

The dataset generated and analyzed during the current study including the holotypes of the new species are available at https://doi.org/10.1594/ PANGAEA.874119.

\section{Publisher's Note}

Springer Nature remains neutral with regard to jurisdictional claims in published maps and institutional affiliations.

Received: 6 December 2016 Accepted: 19 June 2017

Published online: 30 June 2017

\section{References}

1. Appeltans $W$, et al. The magnitude of global marine species diversity. Curr Biol. 2012;22:2189-202.

2. Armonies W, Hellwig-Armonies M. Synoptic patterns of meiofaunal and macrofaunal abundances and specific composition in littoral sediments. Helgoländer Meeresun. 1987;41:83-111.

3. Armonies W, Reise K. Faunal diversity across a sand shore. Mar Ecol-Prog Ser. 2000;196:49-57.

4. Artois T, Fontaneto D, Hummon WD, Mclnnes SJ, Todaro MA, Sørensen MV, Zullini A. Ubiquity of microscopic animals? Evidence from the morphological approach in species identification. In: Fontaneto D, editor. Biogeography of microscopic organisms: Is everything small everywhere?. Cambridge: Cambridge University Press; 2011. p. 244-83.

5. Ax P. Neue Pogaina-Arten (Turbellaria, Dalyellioida) mit Zooxanthellen aus dem Mesopsammal der Nordsee- und Mittelmeerküste. Mar Biol. 1970;5:337-40.

6. Ax P. Zur Systematik und Phylogenie der Trigonostominae (Turbellaria, Neorhabdocoela). Mikrofauna Meeresboden. 1971:4:1-84.

7. Ax P. Plathelminthes aus Brackgewässern der Nordhalbkugel. Stuttgart: Steiner; 2008.

8. Ax P, Heller R. Neue Neorhabdocoela (Turbellaria) vom Sandstrand der Nordsee-Insel Sylt. Mikrofauna Meeresboden. 1970;2:1-46.

9. Bartsch I, Schmidt P. Zur Verbreitung und Ökologie einiger Halacaridae (Acari) in Sandstränden der Ostsee (Kieler Bucht), der Nordsee (Sylt) und des Europäischen Nordmeeres (Tromsö). Mikrofauna Meeresboden. 1979;74:1-37

10. Blome D. Zur Systematik von Nematoden aus dem Sandstrand der Nordseeinsel Sylt. Mikrofauna Meeresboden. 1974;33:1-25.

11. Blome D. Systematik der Nematoda eines Sandstrandes der Nordseeinsel Sylt. Mikrofauna Meeresboden. 1982;86:1-194.

12. Blome D. Ökologie der Nematoda eines Sandstrandes der Nordseeinsel Sylt. Mikrofauna Meeresboden. 1983;88:1-76

13. Buschbaum C, Lackschewitz D, Reise K. Nonnative macrobenthos in the Wadden Sea ecosystem. Ocean Coast Manage. 2012;68:89-101.

14. Cannon JT, Vellutini BC, Smith J, Ronquist F, Jondelius U, Hejnol A. Xenacoelomorpha is the sister group to Nephrozoa. Nature. 2016:530:89-93.

15. Casu M, Lai T, Sanna D, Cossu P, Curini-Galletti M. An integrative approach to the taxonomy of the pigmented European Pseudomonocelis Meixner, 1943 (Platyhelminthes: Proseriata). Biol J Linn Soc. 2009;98:907-22.
16. Coull BC. Ecology of the marine meiofauna. In: Higgins RP, Thiel H, editors. Introduction to the study of meiofauna. Washington: Smithsonian Institution Press; 1988. p. 18-38.

17. Dörjes J. Die Acoela (Turbellaria) der Deutschen Nordseeküste und ein neues System der Ordnung. Z Zool Syst Evol. 1968;6:56-452.

18. Dörjes J. Zur Ökologie der Acoela (Turbellaria) in der Deutschen Bucht. Helgoland Wiss Meer. 1968;18:78-115.

19. Ehlers U. Systematisch-phylogenetische Untersuchungen an der Familie Solenopharyngidae (Turbellaria, Neorhabdocoela). Mikrofauna Meeresboden. 1972;11:1-78

20. Ehlers U. Zur Populationsstruktur interstitieller Typhloplanoida und Dalyellioida (Turbellaria, Neorhabdocoela). Mikrofauna Meeresboden. 1973;19:1-105

21. Ehlers U. Interstitielle Typhloplanoida (Turbellaria) aus dem Litoral der Nordseeinsel Sylt. Mikrofauna Meeresboden. 1974;49:1-102.

22. Ehlers U, Müller B, Franke M. Three new species of the Typhloplanoida (Plathelminthes, Rhabdocoela) from the island of Sylt (North Sea). Microfauna Marina. 1994:9:313-32.

23. Ehlers U, Sopott-Ehlers B. Interstitielle Fauna von Galapagos. XXXVIII. Haloplanella Luther und Pratoplana Ax (Typhloplanoida, Plathelminthes). Microfauna Marina. 1989;5:189-206.

24. Faasse M. The North American ostracod Eusarsiella zostericola (Cushman, 1906) arrives in mainland Europe. Biolnvasions Records. 2013;2:47-50.

25. Faubel A. Die Acoela (Turbellaria) eines Sandstrandes der Nordseeinsel Sylt. Mikrofauna Meeresboden. 1974;32:1-58.

26. Faubel A. Macrostomida (Turbellaria) von einem Sandstrand der Nordseeinsel Sylt. Mikrofauna Meeresboden. 1974:45:1-32.

27. Faubel A. Eine neue Art der Gattung Retronectes (Turbellaria, Catenulida) aus dem Küstengrundwasser der Nordseeinsel Sylt. Zool Scr. 1976;5:217-20

28. Faubel A. Interstitielle Acoela (Turbellaria) aus dem Litoral der nordfriesischen Inseln Sylt und Amrum (Nordsee). Mitt Hamburg Zool Mus Inst. 1976;73:17-56

29. Faubel A. Populationsdynamik und Lebenszyklen interstitieller Acoela und Macrostomida (Turbellaria). Mikrofauna Meeresboden. 1976;56:1-107.

30. Faubel A, Gollasch S. Cryptostylochus hullensis sp. nov. (Polycladida, Acotylea, Platyheltminthes): a possible case of transoceanic dispersal on a ship's hull. Helgoländer Meeresun. 1996;50:533-7.

31. Faubel A, Warwick RM. The marina flora and fauna of the Isles of Scilly: Free-living Plathelminthes ('Turbellaria'). J Nat Hist. 2005;39:1-45.

32. Gerlach SA. Means of meiofauna dispersal. Mikrofauna Meeresboden. 1977;61:89-103.

33. den Hartog C. A preliminary revision of the Proxenetes group (Trigonostomidae, Turbellaria). VIII. P K Ned Akad C Biol. 1966;69:128-38.

34. Hartwig E. Die Ciliaten des Gezeiten-Sandstrandes der Nordseeinsel Sylt. II. Ökologie. Mikrofauna Meeresboden. 1973;21:1-171.

35. Hartwig E. Die Ciliaten des Gezeiten-Sandstrandes der Nordseeinsel Sylt. I. Systematik. Mikrofauna Meeresboden. 1973;18:1-69.

36. Hellwig M. Ökologie freilebender Plathelminthen im Grenzraum WattSalzwiese lenitischer Gezeitenküsten. Microfauna Marina. 1987:3:157-248.

37. Higelke B. Morphodynamik des Lister Tidebeckens. In: Gätje C, Reise K, editors. Ökosystem Wattenmeer-Austausch-, Transport- und Stoffumwandlungsprozesse. Berlin: Springer; 1998. p. 103-26.

38. Horvath TG, Whitman RL, Last LL. Establishment of two invasive crustaceans (Copepoda: Harpacticoida) in the nearshore sands of Lake Michigan. Can J Fish Aquat Sci. 2001:58:1261-4.

39. Hoxhold S. Populationsstruktur und Abundanzdynamik interstitieller Kalyptorhynchia (Turbellaria, Neorhabdocoela). Mikrofauna Meeresboden. 1974:41:1-134.

40. Karling TG. Zur Kenntnis der Gattung Coelogynopora Steinboeck (Turbellaria Proseriata). Ark Zool. 1958;11:559-68.

41. Kossmagk-Stephan K. Marine oligochaeta from a sandy beach of the island of Sylt (North Sea) with description of four new enchytraeid species. Mikrofauna Meeresboden. 1983;89:1-28.

42. Kröncke I, Reiss H, Eggleton JD, et al. Changes in North Sea macrofauna communities and species distribution between 1986 and 2000. Estuar Coast Shelf S. 2011:94:1-15.

43. Luther A. Die Turbellarien Ostfennoskandiens. I. Acoela, Catenulida, Macrostomida, Lecithoepitheliata, Prolecithophora, und Proseriata. Fauna Fennica. 1960;7:1-155. 
44. Martens P, Curini-Galletti MC. Taxonomy and phylogeny of the Archimonocelididae Meixner, 1938 (Platyhelminthes, Proseriata). Bijdr Dierkd. 1993;63:65-102.

45. Menn I, Armonies W. Predatory Promesostoma species (Plathelminthes, Rhabdocoela) in the Wadden Sea. J Sea Res. 1999:41:309-20.

46. Meineke T, Westheide W. Gezeitenabhängige Wanderungen der Interstitialfauna in einem Sandstrand der Insel Sylt (Nordsee). Mikrofauna Meeresboden. 1979;75:1-36.

47. Meixner J (1938) Turbellaria (Strudelwürmer). I. Allgemeiner Teil. In: G Grimpe, E Wagler (eds) Tierwelt der Nord- und Ostsee IV.b.

48. Mielke W. Zwei neue Harpacticoidea (Crustacea) aus dem Eulitoral der Nordseeinsel Sylt. Mikrofauna Meeresboden. 1973;17:1-14.

49. Mielke W. Systematik der Copepoda eines Sandstrandes der Nordseeinsel Sylt. Mikrofauna Meeresboden. 1975;52:1-134.

50. Mielke W. Ökologie der Copepoda eines Sandstrandes der Nordseeinsel Sylt. Mikrofauna Meeresboden. 1976;59:1-86

51. Mock H. Ototyphlonemertes pallida (Keferstein, 1862). Mikrofauna Meeresboden. 1978;67:1-14.

52. Mock H. Chaetonotoidea (Gastrotricha) der Nordseeinsel Sylt. Mikrofauna Meeresboden. 1979;78:1-107.

53. Mock H. Zur Morphologie von Prostomatella arenicola (Hoplonemertini, Monostilifera). Helgoländer Meeresun. 1981:34:491-6.

54. Mock H. Zur Kenntnis von Diurodrilus subterraneus (Polychaeta, Dinophillidae) aus dem Sandhang der Nordseeinsel Sylt. Helgoländer Meeresun. 1981:43:329-35.

55. Müller U, Ax P. Gnathostomulida von der Nordseeinsel Sylt mit Beobachtungen zur Lebensweise und Entwicklung von Gnathostomula paradoxa Ax. Mikrofauna Meeresboden. 1971;9:4-41

56. Noldt U, Wehrenberg C. Quantitative extraction of living Plathelminthes from marine sands. Mar Ecol Prog Ser. 1984;20:193-201.

57. Pawlak R. Zur Systematik und Ökologie (Lebenszyklen, Populationsdynamik) der Turbellarien Gattung Paromalostomum. Helgoland Wiss Meer 1969:19:417-54.

58. Potel P, Reise K. Gastrotricha Macrodasyoida of intertidal and subtidal sandy sediments in the northern Wadden Sea. Microfauna Marina. 1987;3:363-76.

59. Radziejewska T, Gruszka P, Rokicka-Praxmajer J. A home away from home: a meiobenthic assemblage in a ship's ballast water tank sediment. Oceanologia. 2006:48:259-65.

60. Remane A. Verteilung und Organisation der benthonischen Mikrofauna der Kieler Bucht. Wiss Meeresunters. Kiel. 1933;21:161-221.

61. Reise K. Biotic enrichment of intertidal sediments by experimental aggregates of the deposit-feeding bivalve Macoma balthica. Mar Ecol-Prog Ser. 1983;12:229-36.

62. Reise K. Free-living Platyhelminthes (Turbellaria) of a marine sand flat: an ecological study. Microfauna Marina. 1984;1:1-62.

63. Reise K. Coastal change in a tidal backbarrier basin of the northern Wadden Sea: Are tidal flats fading away? Senck Marit. 1998;29:121-7.

64. Reise K, Ax P. A meiofaunal 'Thiobios' limited to the anaerobic sulfide system of marine sand does not exist. Mar Biol. 1979;54:225-37.

65. Rieger RM. Bradynectes sterreri gen. nov., spec. nov., eine neue psammobionte Macrostomide (Turbellaria). Zool Jahrb Syst. 1971;98:205-35.

66. Scherer B. Annual dynamics of a meiofauna community from the 'Sulfide Layer' of a North Sea sand flat. Microfauna Marina. 1985;2:117-61.

67. Schilke K. Kalyptorhynchia (Turbellaria) aus dem Eulitoral der deutschen Nordseeküste. Helgoland Wiss Meer. 1970;21:143-265.

68. Schmidt P. Die quantitative Verteilung und Populationsdynamik des Mesopsammons am Gezeiten-Sandstrand der Nordseeinsel Sylt. I. Faktorengefüge und biologische Gliederung des Lebensraums. Int Revue ges Hydrobiol. 1968;53:723-79.

69. Schmidt P. Die quantitative Verteilung und Populationsdynamik des Mesopsammons am Gezeiten-Sandstrand der Nordseeinsel Sylt. II. Quantitative Verteilung und Populationsdynamik einzelner Arten. Int Revue ges Hydrobiol. 1969;54:95-174.

70. Schmidt P. Zonation of the interstitial polychaete Stygocapitella subterranea (Stygocapitellidae) in European sandy beaches. Mar Biol. 1970;7:319-23.

71. Schmidt P, Teuchert G. Quantitative Untersuchungen zur Ökologie der Gastrotrichen im Gezeiten-Sandstrand der Insel Sylt. Mar Biol. 1969:4:4-23.
72. Sluys R, Faubel A, Rajagopal S, van der Velde G. A new and alien species of "oyster leech" (Platyhelminthes, Polycladida, Stylochidae) from the brackish North Sea Canal, The Netherlands. Helgol Mar Res. 2005;59:310-4.

73. Sopott B. Systematik und Ökologie von Proseriaten (Turbellaria) der deutschen Nordseeküste. Mikrofauna Merresboden. 1972;13:1-72.

74. Sopott B. Jahreszeitliche Verteilung und Lebenszyklen der Proseriata (Turbellaria) eines Sandstrandes der Nordseeinsel Sylt. Mikrofauna Meeresboden. 1973;15:1-106.

75. Sopott-Ehlers B. Coelogynopora visurgis nov. spec. (Proseriata) und andere freilebende Plathelminthes mariner Herkunft aus Ufersanden der Weser. Microfauna Marina. 1989:5:87-93.

76. Sterrer W. Plate tectonics as a mechanism for dispersal and speciation in interstitial sand fauna. Neth J Sea Res. 1973;7:200-22.

77. Sun B, Fleeger JW. Spatial and temporal patterns of dispersion in meiobenthic copepods. Mar Ecol-Prog Ser. 1991;71:1-11.

78. Tzschaschel G. Marine Rotatoria aus dem Interstitial der Nordseeinsel Sylt. Mikrofauna Meeresboden. 1979;71:1-64.

79. Tzschaschel G. Verteilung, Abundanzdynamik und Biologie mariner interstitieller Rotatoria. Mikrofauna Meeresboden. 1980;81:1-56.

80. Uhlig G. Eine einfache Methode zur Extraktion der vagilen mesopsammalen Mikrofauna. Helgoland Wiss Meer. 1964;11:178-85.

81. Weinert M, Mathis M, Kröncke I, Neumann H, Pohlmann T, Reiss H. Modelling climate change effects on benthos: Distributional shifts in the North Sea from 2001 to 2099. Estuar Coast Shelf S. 2016;175:157-68.

82. Westblad E. Marine "Alloeocoels" (Turbellaria) from North Atlantic and Mediterranean coasts.I. Ark Zool. 1955;7:491-526.

83. Westheide W. Parapodrilus psammophilus nov. gen. nov. spec., eine neue Polychaeten-Gattung aus dem Mesopsammal der Nordsee. Helgoland Wiss Meer. 1965;12:207-13.

84. Westheide W. Zur Polychaetenfauna des Eulitorals der Nordseeinsel Sylt. Helgoland Wiss Meer. 1966;13:203-9.

85. Westheide W. Die Gattung Trilobodrilus (Archiannelida, Polychaeta) von der deutschen Nordseeküste. Helgoland Wiss Meer. 1967:16:207-15.

86. Westheide W. Monographie der Gattungen Hesionides Friedrich und Microphthalmus Mecznikow (Polychaeta, Hesionidae). Ein Beitrag zur Organisation und Biologie psammobionter Polychaeten. Z Morphol Tiere. 1967;61:1-159.

87. Willems WR, Artois TJ, Backeljau T, Schockaert ER. Typhloplanoida (Platyhelminthes, Rhabdocoela) from New Caledonia and eastern Australia, with the description of six new taxa. New Zealand J Zool. 2005:32:79-98.

88. Zeppilli D, Sarrazin J, Leduc D, et al. Is the meiofauna a good indicator for climate change and anthropogenic impacts? Mar Biodiv. 2015:45:505-35. doi:10.1007/s12526-015-0359-z

\section{Submit your next manuscript to BioMed Central and we will help you at every step:}

- We accept pre-submission inquiries

- Our selector tool helps you to find the most relevant journal

- We provide round the clock customer support

- Convenient online submission

- Thorough peer review

- Inclusion in PubMed and all major indexing services

- Maximum visibility for your research

Submit your manuscript at www.biomedcentral.com/submit 